\title{
Alternative Models for Moment Inequalities
}

\section{Citation}

Pakes, A. 2010. Alternative Models for Moment Inequalities. Econometrica 78, no. 6: 1783-1822. doi:10.3982/ecta7944

\section{Published Version}

doi:10.3982/ecta7944

\section{Permanent link}

http://nrs.harvard.edu/urn-3:HUL.InstRepos:34708519

\section{Terms of Use}

This article was downloaded from Harvard University's DASH repository, and is made available under the terms and conditions applicable to Other Posted Material, as set forth at http:// nrs.harvard.edu/urn-3:HUL.InstRepos:dash.current.terms-of-use\#LAA

\section{Share Your Story}

The Harvard community has made this article openly available.

Please share how this access benefits you. Submit a story.

Accessibility 


\title{
ECONOMETRICA
}

JOURNAL OF THE ECONOMETRIC SOCIETY

An International Society for the Advancement of Economic Theory in its Relation to Statistics and Mathematics

http://www.econometricsociety.org/

Econometrica, Vol. 78, No. 6 (November, 2010), 1783-1822

\section{ALTERNATIVE MODELS FOR MOMENT INEQUALITIES}

\author{
A. PAKES
}

Harvard University, Cambridge, MA 02138, U.S.A. and National Bureau

of Economic Research

The copyright to this Article is held by the Econometric Society. It may be downloaded, printed and reproduced only for educational or research purposes, including use in course packs. No downloading or copying may be done for any commercial purpose without the explicit permission of the Econometric Society. For such commercial purposes contact the Office of the Econometric Society (contact information may be found at the website http://www.econometricsociety.org or in the back cover of Econometrica). This statement must be included on all copies of this Article that are made available electronically or in any other format. 


\title{
ALTERNATIVE MODELS FOR MOMENT INEQUALITIES
}

\begin{abstract}
BY A. PAKES ${ }^{1}$
Behavioral choice models generate inequalities which, when combined with additional assumptions, can be used as a basis for estimation. This paper considers two sets of such assumptions and uses them in two empirical examples. The second example examines the structure of payments resulting from the upstream interactions in a vertical market. I then mimic the empirical setting for this example in a numerical analysis which computes actual equilibria, examines how their characteristics vary with the market setting, and compares them to the empirical results. The final section uses the numerical results in a Monte Carlo analysis of the robustness of the two approaches to estimation to their underlying assumptions.
\end{abstract}

KEYWORDS: Moment inequalities, behavioral models, structural and reduced forms.

BEHAVIORAL CHOICE MODELS generate inequalities which, when combined with additional assumptions, can be used as a basis for estimation. This paper considers two sets of assumptions which suffice and uses them in two examples which have been difficult to analyze empirically. In doing so, we distinguish between the assumptions needed to estimate the "structural" parameters defined by the primitives of the choice problem and the "reduced form" coefficients obtained from regressing profits on variables of interest.

I begin with a single agent discrete choice problem: a consumer's decision of which supermarket to shop at. This provides a transparent setting to illustrate the assumptions underlying alternative estimators and motivates the more formal discussion in the rest of the paper. The difficulty in analyzing this example arises from the size of its choice set: all possible bundles of goods at "nearby" locations. Its importance stems from the need to analyze similar problems to understand the implications of alternative local policies (zoning laws, public transportation alternatives, and the like).

Section 2 of the paper formalizes two sets of assumptions that take one from the choice model to an estimation algorithm. This is done in a multiple agent setting (with the single agent simplifications noted). The first approach is labeled the generalized discrete choice approach, as it generalizes familiar discrete choice theory to allow for multiple interacting agents. The ideas behind this approach date to Tamer (2003), and are developed in more detail in papers by Ciliberto and Tamer (2009) and Andrews, Berry, and Jia (2006). It was

\footnotetext{
${ }^{1}$ This paper is a revised version of part of my Fisher-Schultz Lecture presented at the World Congress of the Econometric Society in London, August 2005. The paper draws extensively from past interactions with my students and co-authors, and I would like to take this opportunity to express both my intellectual debt and my thanks to them. I like to think they enjoyed the experience as much as I did, although that might have been harder for the students in the group. For help on this paper, I owe a particular debt to Robin Lee. I also thank three referees and a co-editor for helpful comments.
} 
first considered in the context of analyzing two-stage entry games, but is easily adapted to other multiple agent problems.

The second approach is based on the inequalities generated by the difference between the expected profits from the choice made and those from an alternative feasible choice, so we refer to it as the profit inequality approach. It is preceded by the first order (or Euler) condition estimators for single agent dynamic models provided in Hansen and Singleton (1982) and extended to incorporate transaction costs, and hence inequalities, by Luttmer (1996). The approach considered here is a direct extension of the work in Pakes, Porter, Ho, and Ishii (2006), who provided assumptions that enable us to take "revealed preference" inequalities to data (for related work on revealed preference in demand analysis, see Varian (1982), and in the analysis of auctions, see Haile and Tamer (2003)). The two approaches are not nested and a comparison of their assumptions closes this section.

Section 3 applies the frameworks developed in Section 2 to the analysis of markets in which a small number of sellers interact with a small number of buyers. This is typical of upstream interactions in many vertical markets; markets where sellers remarket the goods they buy to consumers. A difficulty in analyzing them is that the contracts that establish the buyers' costs are typically proprietary, and these costs determine both the prices the buyer charges to consumers and the sellers' investment incentives. Costs are also often proprietary in consumer goods markets. However, because there are many consumers in those markets, we typically assume a Nash equilibrium in price (or quantity) in them. Then the first order conditions from that equilibrium can be used to back out marginal cost. The analogous procedure for vertical markets leads us to moment inequalities: we observe who contracts with whom and ask what features must the buyer's cost functions have for each agent to be doing better under the observed set of contracts than what they could have expected from changing their contracting behavior.

The section begins by extending the empirical work of Ho (2009), which characterizes cost functions in HMO-hospital networks. It shows that her approach can be extended to allow for disturbances that are known to the agents when they make their decisions but not to the econometrician. It then compares the empirical results she obtains to those obtained once we allow for these disturbances. Next we compute equilibria for markets similar to those used in the empirical analysis. The numerical results allow us both to investigate the consistency of the empirical results with those obtained from an equilibrium computation and to engage in a more general examination of the correlates of equilibrium markups.

Section 4 uses the data underlying the numerical results of Section 3 in a Monte Carlo analysis of the two approaches to estimation introduced in Section 2. It focuses on the behavior of the two estimators when one or more of the assumptions needed to derive their properties is violated. At least in our example, the estimators were robust to all assumptions except that on the form 
of the disturbance distribution, an assumption which is required only for the generalized discrete choice model. ${ }^{2}$

\section{A MOTIVATING EXAMPLE}

I begin with a single agent example taken from an unpublished thesis by Michael Katz (2007); I thank him for permission to use it. Katz's goal was to estimate the costs shoppers assign to driving to supermarkets. Transportation costs are central to understanding store location decisions, and hence to the analysis of the impact of regulations (e.g., zoning laws) and policy changes (e.g., public transportation projects) on retail trade. They have been difficult to analyze empirically with traditional discrete choice models because of the size and complexity of the choice set facing consumers (all possible bundles of goods at all nearby stores). In contrast, large choice sets facilitate moment inequality estimators, as they give the empirical researcher a greater ability to chose a counterfactual that is likely to isolate the effect of interest (here the cost of travel time).

Assume that the agents' utility functions are additively separable functions of the utility from the basket of goods the agent buys, expenditure on that basket, and drive time to the supermarket. The agent's decision, say $d_{i}$, consists of buying a basket of goods, say $b_{i}$, at a particular store, say $s_{i}$, so $d_{i}=\left(b_{i}, s_{i}\right)$. If $z_{i}$ represents individual characteristics, $U\left(b_{i}, z_{i}\right)$ and $d t\left(s_{i}, z_{i}\right)$ provide individual $i$ 's utility from $b_{i}$ and drive time to $s_{i}$, respectively, and $e\left(b_{i}, s_{i}\right)$ is the expenditure required to buy $b_{i}$ at $s_{i}$, then the agent's utility from $\left(b_{i}, s_{i}\right)$ is

$$
\pi\left(d_{i}, z_{i}, \theta\right)=U\left(b_{i}, z_{i}\right)-e\left(b_{i}, s_{i}\right)-\theta_{i} d t\left(s_{i}, z_{i}\right)
$$

where I have normalized the coefficient on expenditure to 1 so $\theta_{i}$, the disutility of a unit of drive time, is in dollars.

To proceed using moment inequalities, we need to compare the utility from the choice the individual made to the utility from a choice the individual could have made but chose not to. This is a sample design question, as it determines what variance is used to estimate the parameter. For a particular $d_{i}$, we chose the alternative, say $d^{\prime}\left(d_{i}\right)$, to be the purchase of (i) the same basket of goods, (ii) at a store which is further away from the consumer's home than the store the consumer shopped at. Note that, given the additive separability assumption, this choice differences out the impact of the basket of goods chosen on utility; that is, it allows us to hold fixed the dimension of the choice that is not of direct interest and to investigate the impact of travel time in isolation.

\footnotetext{
${ }^{2}$ Since the theoretical restrictions brought to data are moment inequalities, they typically lead to set valued estimators. Methods of inference for set valued estimators are an active and important area of econometric research that I do not discuss here; see, in particular, Chernozhukov, Hong, and Tamer (2007), Andrews and Soares (2010), and the papers cited above.
} 
Assume the agent makes his choice of store by maximizing his expected utility (equation (1)) conditional on the information at his disposal when he chooses the store to shop at. We denote the agent's expectation operator by $\mathcal{E}(\cdot)$ and his information set by $\mathcal{J}_{i}$. Note that when the agent makes this choice, the goods that will be bought at the store are a random variable, say $\mathbf{b}$, as are their prices and hence total expenditure $\mathbf{e}(\cdot)$. If the bundle bought at the chosen store could have been bought at the alternate store, then $\left(s^{\prime}, b_{i}^{\prime}\right)$, where $b_{i}^{\prime}$ is what would have been bought had the agent gone to $s^{\prime}$, is preferred over $\left(s_{i}^{\prime}, b_{i}\right)$. Since $s_{i}$ was preferred over $s_{i}^{\prime}$, transitivity of preferences insures that if, for any function $f(\cdot), \Delta f\left(d, d^{\prime}, \cdot\right) \equiv f(d, \cdot)-f\left(d^{\prime}, \cdot\right)$

$$
\mathcal{E}\left[\Delta \pi\left(\mathbf{b}, s_{i}, s_{i}^{\prime}, z_{i}\right) \mid \mathcal{J}_{i}\right]=\mathcal{E}\left[-\Delta \mathbf{e}\left(\mathbf{b}, s_{i}, s_{i}^{\prime}\right)-\theta_{i} \Delta d t\left(s_{i}, s_{i}^{\prime}, z_{i}\right) \mid \mathcal{J}_{i}\right] \geq 0 .
$$

We let $\nu_{1, i, s, s^{\prime}}$ be the difference between realized and expected utility, that is,

$$
\nu_{1, i, s, s^{\prime}} \equiv \Delta \pi\left(b, s_{i}, s_{i}^{\prime}, z_{i}\right)-\mathcal{E}\left[\Delta \pi\left(\mathbf{b}, s_{i}, s_{i}^{\prime}, z_{i}\right) \mid \mathcal{J}_{i}\right],
$$

and consider two different assumptions on the distribution of the $\theta_{i}$.

CASE 1: Assume $\theta_{i}=\theta_{0}$ or, more generally, that all determinants of the costs of drive time are observed and incorporated in the econometrician's specification. Letting $\rightarrow_{P}$ denote convergence in probability, then since $\Delta d t\left(s_{i}, s_{i}^{\prime}, z_{i}\right)<0$,

$$
N^{-1} \sum_{i} \nu_{1, i, s, s^{\prime}} \rightarrow_{P} 0 \quad \text { implies }-\frac{\sum_{i} \Delta e\left(b_{i}, s_{i}, s_{i}^{\prime}\right)}{\sum_{i} \Delta d t\left(s_{i}, s_{i}^{\prime}, z_{i}\right)} \rightarrow_{p} \underline{\theta} \leq \theta_{0} .
$$

To obtain an upper bound for $\theta_{0}$, consider an alternative store $\left(s_{i}^{\prime \prime}\right)$ which was closer to the individual. An analogous argument shows

$$
N^{-1} \sum_{i} \nu_{1, i, s, s^{\prime \prime}} \rightarrow_{P} 0 \quad \text { implies } \quad-\frac{\sum_{i} \Delta e\left(b_{i}, s_{i}, s_{i}^{\prime \prime}\right)}{\sum_{i} \Delta d t\left(s_{i}, s_{i}^{\prime \prime}, z_{i}\right)} \rightarrow_{p} \bar{\theta} \geq \theta_{0} .
$$

That is, provided the average of the expectational errors converges to zero, equations (2) and (3) give us asymptotic bounds for $\theta_{0}$.

CASE 2: Now assume that there is a determinant of the cost of drive time that the agent knows but is not observed by the econometrician, that is, $\theta_{i}=$ $\left(\theta_{0}+\nu_{2, i}\right)$, where $\sum \nu_{2, i} \equiv 0$, so $\theta_{0}$ is the mean cost of drive time. Then our inequality becomes

$$
\mathcal{E}\left[\Delta \mathbf{e}\left(\mathbf{b}_{i}, s_{i}, s_{i}^{\prime}\right)-\left(\theta_{0}+\nu_{2, i}\right) \Delta d t\left(s_{i}, s_{i}^{\prime}, z_{i}\right) \mid \mathcal{J}_{i}\right] \geq 0 .
$$


Now assume the agent knows drive time in deciding where to shop, that is, $\Delta d t(\cdot) \in \mathcal{J}_{i}$. Then

$$
\begin{aligned}
& \frac{1}{N} \sum_{i} \Delta d t\left(s_{i}, s_{i}^{\prime}, z_{i}\right)^{-1} \nu_{1, i, s, s^{\prime}} \rightarrow_{P} 0, \quad \text { which implies } \\
& \frac{1}{N} \sum_{i}\left(\frac{\Delta e\left(b_{i}, s_{i}, s_{i}^{\prime}\right)}{\Delta d t\left(s_{i}, s_{i}^{\prime}, z_{i}\right)}\right) \rightarrow_{P} \underline{\theta} \leq \theta_{0} .
\end{aligned}
$$

An analogous upper bound to $\theta_{0}$ is generated by choosing an alternative that has drive time less than that of the chosen store.

\section{Discussion}

Case 1 uses a ratio of averages to bound the parameter of interest, while Case 2 uses the average of a ratio. The following points should be kept in mind.

CASE 1 Versus CASE 2: Case 2 allows for unobserved heterogeneity in the coefficient of interest and does not need to specify what the distribution of that unobservable is. In particular, the unobservable can be freely correlated with the right-hand side variable. "Drive time" is a choice variable, so we might expect it to be correlated with the perceived costs of that time (with $\nu_{2, i}$ ). If it is, then Case 1 and Case 2 estimators should be different; otherwise they should be the same. So there is a test for whether any unobserved differences in preferences are correlated with the "independent" variable, and that test does not require us to specify a conditional distribution for $\nu_{2, i}$. Similar issues arise in analyzing the choice of a durable good when there is an intensity of use decision that follows the choice of the durable (for a classic example, see Dubin and McFadden (1984)).

BEHAVIORAL CONDITIONS: This is a two-stage model with uncertainty: an initial choice of where to shop is made before knowing what prices are, and a choice of what to buy is made after arriving at the store. Note, however, that we did not have to specify either the information on prices the agent had at his disposal when he made his initial decision or the form of the agent's prior price distribution conditional on that information. These are objects econometricians seldom have access to.

Conditions on the ChOice SeT: All we required of the choice set was one feasible alternative. In particular, we did not need to specify and compute returns for the many possible "inside" choices, and we did not need to specify an "outside" alternative.

Finally note that our focus on the drive time coefficient led us to chose an alternative that differenced out any heterogeneity in preferences over bundles 
of goods. If instead we were interested in the utility of a particular good, we would compare baskets with and without that good at the same store. If we had multiple observations on the same individual, there are many more (largely unexplored) possibilities.

\subsection{Estimates From the Inequality and a Comparison Model}

Katz (2007) estimated his model using the Nielsen Homescan Panel, 2004, for household expenditures and data from Retail Site Database of TradeDimensions for the characteristics of stores. He used the shopping trips of about 1,300 families in Massachusetts and surrounding counties and compared the results that use inequalities to the results that he obtained from estimating a discrete choice comparison model.

\section{The Comparison Model}

To obtain the econometric implications of a behavioral model of supermarket choice, we would (i) specify the agent's prior distribution of prices at each store, (ii) compute the bundle of goods the agent would buy for each possible realization of the price vector at the store, and (iii) form the expected utility of going to the store. These are demanding tasks. Similar considerations lead most (though not all; see below) analysis of single agent discrete choice problems to reduced forms. The reduced form can be given an appealing interpretation by constructing it from the regressions of expected returns from each choice on variables of interest. If we then make a sufficiently powerful assumption on the joint distribution of the regression functions disturbances, the functions themselves can be estimated. It is the fact that an analogous reduced form is not useful in multiple agent problems that lead to the generalized discrete choice model considered below.

Unfortunately this reduced form cannot be used in the supermarket choice problem without first reducing the dimension of the choice set. Katz assumed the number of weekly visits made to supermarkets is distributed as a Poisson random variable. At each visit, the consumer chooses between 10 expenditure bundles at each of the outlets within a given radius of his home. The utility function for a given expenditure bundle and store is allowed to differ with the number of shopping trips per week, but for a fixed number of trips is given by equation (1) augmented with an additive "logit" error. The expenditure bundles are constructed from typical purchase patterns for a given amount of expenditure which are then priced at each outlet (giving us the expenditure level for each choice). ${ }^{3}$

There are a number of reasons to doubt the estimates from this model. I focus on those directly related to the price and drive time variables. First, the

\footnotetext{
${ }^{3}$ For a discussion of alternative ways to build reduced forms for supermarket discrete choice problems and an application, see Beckert, Griffith, and Nesheim (2009).
} 
prices for the expenditure class need not reflect the prices of the goods the individual actually is interested in, so there is an error in the price variable, and if individuals shop at stores where the goods they are interested in are less costly, that error is negatively correlated with the price itself. Second, the model does not allow for expectational errors, so agents are assumed to know all relevant prices when store choice decisions were made (and there are a lot of them). Finally, the model does not allow for unobserved heterogeneity in the aversion to drive time. One could allow for a random coefficient on drive time and integrate it out, but this would require an assumption on the conditional distribution for that variable, and given that the aversion to drive time is likely to be related to drive time per se, a traditional random coefficient assumption would be suspect.

\section{Results}

The specifications used for the models estimated were quite detailed; the comparison model estimated about 40 different parameters for each of three different number of visits per week, while the revealed choice model estimated about 15 . Both models were estimated with specifications that included outlet characteristics and interactions between expenditure and demographics, so the aversion to drive time varied with observed individual characteristics. As in the original paper, I focus on the estimates of the median (of the mean) aversion to drive time.

The multinomial comparison models were estimated using maximum likelihood. The median aversion to drive time was estimated at $\$ 240$ per hour. The median wage in the region was $\$ 17$ per hour, so this estimate is implausibly high. Also several of the other coefficients had the "wrong" sign.

The inequality estimators were obtained from differences between the chosen store and four different counterfactual store choices (chosen to reflect price and distance differences with the chosen store). Each comparison was interacted with positive functions of 26 "instruments" (variables that were assumed to be mean independent of the expectational errors), producing over 100 moment inequalities. As is not unusual for problems with many more inequalities than bounds to estimate, the inequality estimation routine generated point (rather than interval) estimates for the coefficients of interest (there was no value of the parameter vector that satisfied all of the moment inequalities). However, tests indicated that one could accept the null that this result was due to sampling error. ${ }^{4}$

\footnotetext{
${ }^{4}$ The finding that there is no value of the parameter vector that satisfies all the inequalities is not unusual in moment inequality problems with many inequalities. Consider the one parameter case. When there are many moment inequalities there are many upper and lower bounds for that parameter. The estimation routine forms an interval estimate from the least upper and the greatest lower bound. The approximate normality of finite sample means implies that in finite samples, the least upper bound will have a negative bias and the greatest lower bound will have a
} 
The inequality estimators that corresponded to Case 1 above, that is, those that did not allow for unobserved heterogeneity in the drive time coefficient, produced median aversions to drive time of about $\$ 4$ per hour. The estimators that corresponded to Case 2 above, the case that did allow for heterogeneity in the drive time coefficient, generated estimates of the median aversion to drive time that varied between $\$ 16$ and $\$ 18$ per hour, depending on the specification. The difference between the two estimators is consistent with there being unobserved heterogeneity in the drive time coefficient that is negatively correlated with drive time itself; a result one would expect from a model where drive time itself was a choice variable. Moreover, in the model which allowed for heterogeneity, the other coefficients took on values which accorded with intuition.

\section{CONDITIONS FOR MOMENT INEQUALITY ESTIMATORS}

This section provides two sets of conditions that can be used to justify moment inequality estimators in more general (both multiple and single agent) settings. For each of the two approaches, we consider estimation of both the parameters of the underlying behavioral model and a reduced form constructed by regressing expected profits on variables of interest. Each approach is defined by four assumptions: two that are common across approaches and two that differ. I begin with the two common assumptions.

\subsection{Common Assumptions}

The first condition is that agents expect their choice to lead to higher returns than alternative feasible choices. Let $\pi(\cdot)$ be the profit function, let $d_{i}$ and $d_{-i}$ be the agent's and his competitors' choices, let $D_{i}$ be the choice set, let $\mathcal{J}_{i}$ be the agent's information set, and let $\mathcal{E}$ be the expectation operator used by the agent to evaluate the implications of his actions.

C1: We assume

$$
\sup _{d \in D_{i}} \mathcal{E}\left[\pi\left(d, \mathbf{d}_{-i}, \mathbf{y}_{i}, \theta_{0}\right) \mid \mathcal{J}_{i}\right] \leq \mathcal{E}\left[\pi\left(d_{i}=d\left(\mathcal{J}_{i}\right), \mathbf{d}_{-i}, \mathbf{y}_{i}, \theta_{0}\right) \mid \mathcal{J}_{i}\right],
$$

where $\mathbf{y}_{i}$ is any variable (other than the decision variables) which affects the agent's profits and the expectation is calculated using the agent's beliefs on the likely values of $\left(\mathbf{d}_{-i}, \mathbf{y}_{i}\right)$. Throughout, variables that the decision maker views as random will be boldface, while realizations of those random variables will be represented by standard typeface.

positive bias. So the two can easily cross. The test is a test of whether such crossings could have been a result of sampling error. 
Three points about $\mathrm{C} 1$ are central to what follows. First, there are no restrictions on either the choice set or the objective function. In particular, the objective function need not be concave in $d, D$ could be discrete (e.g., a choice among bilateral contracts, ordered choice, ...) or continuous (e.g., the choice of the location and size of a retail outlet), and when continuous, $d_{i}$ can be at a corner of the choice set. Second, $\mathrm{C} 1$ is a necessary condition for a best response. As a result, were we to assume a Nash equilibrium, $\mathrm{C} 1$ would be satisfied regardless of the equilibrium selection mechanism. Finally, note that $\mathrm{C} 1$ is meant to be a rationality assumption in the sense of Savage (1954); that is, the agent's choice is optimal with respect to the agent's beliefs. In itself, it does not place any restrictions on the relationship of those beliefs to the data generating process. We will need to restrict beliefs to evaluate estimators, but the restrictions used differ between the two approaches to estimation.

Both approaches require a model capable of predicting what expected profits would be were the agent to deviate from his observed choice. This is the sense in which both require a "structural" model. To predict what expected profits would be from a counterfactual choice, we need to model what the agent thinks that $y_{i}$ and $d_{-i}$ would be were he to change his own decision. For example, consider a two period model to determine the number of outlets $\left(d_{i}\right)$ a retailer builds. Returns from the choice of $d_{i}$ will be a function of postentry prices (which are in $y_{i}$ ) and the number of competing outlets $\left(d_{-i}\right)$. If either of these are likely to change when $d_{i}$ is changed to $d^{\prime}$, we need a model of that change so as to construct the firm's profits from its counterfactual choice. $\mathrm{C} 2$ formalizes this requirement.

We say $\mathbf{y}_{i}$ and/or $\mathbf{d}_{-i}$ are endogenous if they change in response to a change in $d_{i} ; \mathbf{z}_{i}$ will represent a set of exogenous variables, that is, variables whose distributions do not change in response to changes in $d_{i}$. Then our second condition can be stated:

C2: $\mathbf{y}_{i}=y\left(\mathbf{z}_{i}, d, \mathbf{d}_{-i}, \theta\right)$ and $\mathbf{d}_{-i}=d^{-i}\left(d, \mathbf{z}_{i}, \theta\right)$, and the distribution of $\mathbf{z}_{i}$ conditional on $\left(J_{i}, d_{i}=d\right)$ does not depend on $d$.

In words, C2 states that if either $\mathbf{y}_{i}$ or $\mathbf{d}_{-i}$ is endogenous, we need a model for its response to changes in $d_{i}$, and the model must produce a value for the endogenous variable which depends only on decisions and exogenous variables. The condition that the distribution of $\mathbf{z}_{i}$ does not depend on the agent's choice is what we mean when we say that $\mathbf{z}_{i}$ is exogenous.

The restrictiveness of $\mathrm{C} 2$ will vary with the problem. In single agent problems, profits do not depend on $d_{-i}$ and the agent's decision is typically not thought to effect environmental conditions, so $\mathbf{y}_{i}$ is exogenous. Then $\mathrm{C} 2$ is unobjectionable. In multiple agent simultaneous move games $d^{-i}\left(d \neq d_{i}, \mathbf{z}_{i}, \theta\right)=\mathbf{d}_{-i}$, so there is no need for an explicit model of reactions by competitors, but $\mathbf{y}_{i}$ often contains price and/or quantity variables which are endogenous. If, in a sequen- 
tial move game, we want to consider counterfactuals for agents who move early, we need a model for the responses of the agents that move later $\left(\right.$ for $\left.\mathbf{d}_{-i}\right){ }^{5}$

\section{Implications of $C 1$ and $C 2$}

Let $d^{\prime} \in D_{i}$ and define

$$
\begin{aligned}
\Delta \pi\left(d_{i}, d^{\prime}, d_{-i}, z_{i}, \theta_{0}\right) \equiv & \pi\left(d_{i}, d_{-i}, y_{i}, \theta_{0}\right) \\
& -\pi\left(d^{\prime}, d^{-i}\left(d^{\prime}, z\right), y\left(z_{i}, d^{\prime}, d_{-i}\right), \theta_{0}\right) .
\end{aligned}
$$

Then together $\mathrm{C} 1$ and $\mathrm{C} 2$ imply the inequality

$$
\mathcal{E}\left[\Delta \pi\left(d_{i}, d^{\prime}, \mathbf{d}_{-i}, \mathbf{z}_{i}, \theta_{0}\right) \mid \mathcal{J}_{i}\right] \geq 0 \quad \forall d^{\prime} \in D_{i} .
$$

To move from (5) to a moment inequality we can use in estimation requires the following:

- We need a measurement model which determines the relationship between the $\pi(\cdot, \theta)$ and $\left(z_{i}, d_{i}, d_{-i}\right)$ that appear in the theory and the measures of them we use in estimation.

- We need the relationship between the expectation operator underlying the agents decisions (our $\mathcal{E}(\cdot)$ ) and the sample moments that the data generating process provides.

These are the two aspects of the problem which differ across the two approaches. We begin with a measurement model which nests both their assumptions.

\subsection{Measurement Model}

Let $r\left(d, d_{-i}, z^{o}, \theta\right)$ be the profit function specified by the econometrician up to an additively separable disturbance (so the $z^{o}$ are observed), and define $\nu(\cdot$ ) to be the difference between the profit function the agent responds to and this specification, so that

$$
r\left(d, d_{-i}, z_{i}^{o}, \theta\right) \equiv \pi\left(d, d_{-i}, z_{i}, \theta\right)+\nu\left(d, d_{-i}, z_{i}^{o}, z_{i}, \theta\right) .
$$

The agent's decision is based on $\mathcal{E}\left[\pi(\cdot) \mid \mathcal{J}_{i}\right]$. We observe $r(\cdot)$ and have constructed $\nu$ so that $\mathcal{E}\left[r(\cdot) \mid \mathcal{J}_{i}\right]=\mathcal{E}\left[\pi(\cdot) \mid \mathcal{J}_{i}\right]+\mathcal{E}\left[\nu(\cdot) \mid \mathcal{J}_{i}\right]$. It follows that

$$
r\left(d, d_{-i}, z_{i}^{o}, \theta\right) \equiv \mathcal{E}\left[\pi\left(d, \mathbf{d}_{-i}, \mathbf{z}_{i}, \theta\right) \mid \mathcal{J}_{i}\right]+\nu_{2, i, d}+\nu_{1, i, d},
$$

\footnotetext{
${ }^{5}$ Often it is natural to write that model recursively, so that each agent's decision depends on the decisions of the agents who move prior to it. The fact that we allow for sequential games explains the difference between our C2 and Assumption 2 in Pakes et al. (2006). The buyer-seller network example in the next section is sequential and illustrates the types of assumptions needed to model $\mathbf{d}_{-i}$.
} 
where

$$
\nu_{2, i, d} \equiv \mathcal{E}\left[\nu\left(d, \mathbf{d}_{-i}, z_{i}^{o}, \mathbf{z}_{i}, \theta\right) \mid \mathcal{J}_{i}\right]
$$

and

$$
\nu_{1, i, d} \equiv\left(\pi(d, \cdot)-\mathcal{E}\left[\pi(d, \cdot) \mid \mathcal{J}_{i}\right]\right)+\left(\nu(d, \cdot)-\mathcal{E}\left[\nu(d, \cdot) \mid \mathcal{J}_{i}\right]\right) .
$$

Equation (7) expresses the difference between the researcher's specification of profits (i.e., $r(\cdot))$ and the function the agent bases its decision on (i.e., $\left.\mathcal{E}\left[\pi(\cdot) \mid J_{i}\right]\right)$ as a sum of three components, two of which I have grouped together into $\nu_{1}$. The grouping was done because, when evaluated at $\theta=\theta_{0}$, they both are "mean independent" of $\mathcal{J}_{i}$ under the agent's expectation operator (under $\mathcal{E}$ ) by construction. $\nu_{2}$ does not share this property and it is this distinction which forces us to keep track of two separate disturbances below. The relative importance of the two disturbances will differ with the application.

\section{Sources of $\nu_{1}$}

$\nu_{1}$ is a sum of two terms. $\pi(d, \cdot)-\mathcal{E}\left[\pi(d, \cdot) \mid \mathcal{J}_{i}\right]$ provides the difference between the agent's expectation of profits at the time he makes his decision and the realization of profits. In single agent problems, it is solely a result of uncertainty in the exogenous variables whose realizations help determine returns (in the supermarket example, the uncertainty in the prices). In multiple agent problems, there may also be uncertainty in $\mathbf{d}_{-i}$. In either case, to compute the distribution of $\pi(d, \cdot)-\mathcal{E}\left[\pi(d, \cdot) \mid \mathcal{J}_{i}\right]$, we would have to specify the probabilities each agent assigns to different outcomes conditional on their information sets (objects we often know little about). To compute the distribution of $\nu_{1}$ in the multiple agent case, we would also have to solve for an equilibrium conditional on all possible realizations of $\mathbf{d}_{-i}$. This would both be computationally burdensome and require an assumption that selects among possible equilibria. The second component of $\nu_{1}, \nu(d, \cdot)-\mathcal{E}\left[\nu(d, \cdot) \mid \mathcal{J}_{i}\right]$, results either from measurement error in observables or from a specification error in $r(\cdot)$ that is mean independent of $\mathcal{J}_{i}$. Note that such a "specification" error occurs when $r(\cdot)$ is formed by regressing the true profit function onto variables of interest to obtain a "reduced form" whose coefficients become the focus of investigation.

\section{Sources of $\nu_{2}$}

$\nu_{2}$ is defined to equal that part of profits that the agent can condition on when he makes his decisions but the econometrician does not include in the specification. So although it is not known to the econometrician, $\nu_{2, i} \in \mathcal{J}_{i}$, and since $d_{i}=d\left(\mathcal{J}_{i}\right), d_{i}$ will generally be a function of $\nu_{2, i}$. In the supermarket example, $\nu_{2, i}$ has two components: the utility from the goods bought (the $U\left(b_{i}, z_{i}\right)$ in equation (1)), and, in Case 2, the differences between the individual's and the average drive time coefficients $\left(\theta_{i}-\theta_{0}\right)$. In multiple agent problems, $d_{i}$ might also be a function of $\nu_{2,-i}$. 


\section{Selection}

We can now explain the selection problem in behavioral models. Assume that $x$ is an "instrument" in the sense that $\mathcal{E}\left[\nu_{2} \mid x\right]=0$ and, in addition, that $x \in \mathcal{J}$. Then

$$
\mathcal{E}\left[\nu_{1} \mid x\right]=\mathcal{E}\left[\nu_{2} \mid x\right]=0 .
$$

These expectations do not, however, condition on the decision actually made (our $d_{i}$ ), and any moment which depends on the selected choice requires properties of the disturbance conditional on the $d_{i}$ the agent selected. Since $d_{i}$ is measurable $\sigma\left(\mathcal{J}_{i}\right)$ and $\nu_{1}$ is mean independent of any function of $\mathcal{J}$,

$$
\mathcal{E}\left[\nu_{1, i, d} \mid x_{i}, d_{i}\right]=0 \text {, however } \mathcal{E}\left[\nu_{2, i, d} \mid x_{i}, d_{i}\right] \neq 0 .
$$

As a result, the covariance of $x$ and the residuals will typically not be zero when $\theta=\theta_{0}$, the condition we generally require of an "instrument."

To see why $\mathcal{E}\left[\nu_{2, i} \mid x_{i}, d_{i}\right] \neq 0$, consider a single agent binary choice problem $\left(d_{i} \in\{0,1\}\right)$. Then $d_{i}=1$ implies

$$
\mathcal{E}\left[\Delta \pi\left(d_{i}=1, d^{\prime}=0, \cdot\right) \mid \mathcal{J}_{i}\right]=\mathcal{E}\left[\Delta r\left(d_{i}=1, d^{\prime}=0, \cdot\right) \mid \mathcal{J}_{i}\right]+\Delta \nu_{2, i} \geq 0,
$$

where $\Delta \nu_{2, i}=\nu_{2, i, d=1}-\nu_{2, i, d=0}$. So for every agent with $d_{i}=1$,

$$
\Delta \nu_{2, i}>-\mathcal{E}\left[\Delta r\left(d_{i}=1, d^{\prime}=0, \cdot\right) \mid \mathcal{J}_{i}\right] .
$$

If $x_{i}$ is correlated with $\mathcal{E}\left[\Delta r\left(d_{i}=1, d^{\prime}=0, \cdot\right) \mid \mathcal{J}_{i}\right]$, and if $x$ is used as an instrument it is likely to be correlated $\Delta \pi(\cdot)$, then the expectation of $\Delta \nu_{2, i}$ given $x_{i}$ and $d_{i}=1$ will be not be zero, regardless of whether $\mathcal{E}\left[\nu_{2, i, d=1} \mid x_{i}\right]=$ $\mathcal{E}\left[\nu_{2, i, d=0} \mid x_{i}\right]=0$. In words, if $d_{i}$ was selected, then the difference in the unobservable part of the incremental expected returns to $d_{i}$ must have been greater than the (negative of the) difference in the observable part of the incremental returns, and the latter will typically be correlated with our instruments.

\subsection{The Generalized Discrete Choice Approach}

The measurement model in equation (7) provides the notation needed to clarify the conditions needed to move from the profit inequalities in equation (5) to sample moments for our two approaches. Recall that we also need to clarify the relationship between the agent's perceptions of expected returns and the returns emanating from the data generation process embedded in those approaches. We begin with the generalized discrete choice approach (the approach originally developed to handle entry games in the papers by Tamer (2003), Ciliberto and Tamer (2009), and Andrews, Berry, and Jia (2006)).

The multiple agent versions of the generalized discrete choice approach make the following assumption: 
DC3: $\forall d \in D_{i}$,

$$
\pi\left(d, d_{-i}, z_{i}, \theta_{0}\right)=\mathcal{E}\left[\pi\left(d, \mathbf{d}_{-i}, \mathbf{z}_{i}, \theta_{0}\right) \mid \mathcal{J}_{i}\right],
$$

or that there is no uncertainty in either the exogenous variables (in $\mathbf{z}_{i}$ ) or in the actions of the firm's competitors (in $\mathbf{d}_{-i}$ ).

Together C1 and DC3 imply that agents never err. ${ }^{6}$

It is important to note that there are parts of the single agent discrete choice literature that $d o$ allow for uncertainty. These include both the dynamic single agent discrete choice models that explicitly account for randomness in exogenous variables and the literature that uses survey data on expectations in conjunction with choice models to allow for uncertainty (see Keane and Wolpin (2009) and Manski (2004), respectively, and the literature they cite). However, computational difficulties and a lack of information on agents' perceptions on the likely behavior of their competitors have made it difficult to use analogous techniques in multiple agent problems.

DC4 provides the restrictions the generalized discrete choice model places on the measurement model in equation (7).

DC4: $\forall d \in \mathcal{D}_{i}, r\left(d, d_{-i}, z_{i}^{o}, \theta\right)=\pi\left(d, d_{-i}, z_{i}, \theta\right)+\nu_{1, i}$ for a known $\pi(\cdot, \theta)$ and $z_{i}=\left(\left\{\nu_{2, i, d}\right\}_{d}, z_{i}^{o}\right)$ with $\left.\left(\nu_{2, i, d}, \nu_{2,-i, d}\right)\right|_{d, z_{i}^{o}, z_{-i}^{o}} \sim F(\cdot ; \theta)$ for a known $F(\cdot, \theta)$.

The first line in DC4 states that there are no decision specific errors in our profit measure $\left(\nu_{1, i}\right.$ does not have a $d$ subscript). So if we knew $\left(d_{i}, d_{-i}, z_{i}, z_{-i}\right)$, we could construct an exact measure of profit differences for each $\theta$. The second line states that $z_{i}$ has both observed (the $z_{i}^{o}$ ) and unobserved (the $\nu_{2, i, d}$ ) components, and provides their properties. The distribution of the unobserved conditional on the observed components is known up to a parameter vector, ${ }^{7}$ and there is no measurement error in the observed components. Since DC3 assumes full information, all the $\nu_{2, i, d}$ are known to all agents when decisions are made, just not to the econometrician. Note that given DC3, the distribution $F(\cdot \mid \cdot)$ appearing in DC4 is a distribution of realized values, and hence must be consistent with the data generating process (more on this below).

Although the assumptions used in DC3 and DC4 may seem restrictive, they clearly advanced the study of discrete choice models in multiple agent settings. Recall that single agent discrete choice models can always be given an intuitive

\footnotetext{
${ }^{6}$ As stated, DC3 also rules out the analysis of sequential games in which an agent who moves initially believes that the decisions of an agent who moves thereafter depend on his initial decision. However, with only notational costs, we could allow for a deterministic relationship between a component of $d_{-i}$ and $(d, z)$.

${ }^{7}$ There are papers in the single agent discrete choice literature which have allowed for classification errors in $d$; see, for example, Hausman, Abrevaya, and Scott-Morton (1998). At least in principle, such errors could be added to any of the models considered here.
} 
reduced form interpretation. Simply regress expected returns from alternate decisions onto observed variables and the decision itself, then solve for the optimal $d$ conditional on the observables and the disturbances from the regressions, and finally make an assumption on the joint distribution of those disturbances that enables identification. The analogous reduced form for multiple agent problems proved not to be useful. In multiple agent contexts, researchers were interested in the relationship between profits and $\left(d_{-i}, z\right)$ conditional on unobservable determinants of profits, particularly those that were correlated with $d_{-i}$. For example, in the entry models that stimulated this literature, there was a focus on the relationship of profitability to the number of entrants. Models which did not allow for unobserved market characteristics that affected the profitability of all potential entrants in a market often estimated coefficients that implied that a firm's profits increased in the number of competitors (since more profitable markets attracted more entrants). So to provide a reduced form of interest for the relationship between profits and $\left(z_{i}, d_{i}, d_{-i}\right)$, we needed to allow for a disturbance that was correlated with $d_{-i}$. This is the problem the generalized discrete choice approach sought to solve.

Substituting DC3 and DC4 into the model generated by $\mathrm{C} 1$ and $\mathrm{C} 2$ (equation (5)), and letting $\nu_{2, i} \equiv\left\{\nu_{2, i, d}\right\}_{d}$, with analogous notation for $\nu_{2,-i}$, we obtain the following model:

MODEL D: $\forall d^{\prime} \in D_{i}$,

$$
\Delta \pi\left(d_{i}, d^{\prime}, d_{-i}, z_{i}^{o}, \nu_{2, i} ; \theta_{0}\right) \geq 0 ;\left.\quad\left(\nu_{2, i}, \nu_{2,-i}\right)\right|_{z_{i}^{o}, z_{-i}^{o}} \sim F\left(\cdot ; \theta_{0}\right) .
$$

To insure that there exists a $\theta$ for which the event $\Delta \pi\left(d_{i}, d^{\prime}, d_{-i}, z_{i}^{o}, \nu_{2, i}\right.$; $\theta) \geq 0$ has positive probability $\forall d \in D_{i}$ and all agents in each market, we need further conditions on $F(\cdot)$ and/or $\pi(\cdot)$. The additional restriction typically imposed is that the profit function is additively separable in the unobserved determinants of profits:

$$
\mathrm{RD}^{\text {as: }}: \forall d \in D_{i},
$$

$$
\pi\left(d, d_{-i}, z_{i}^{o}, \nu_{2, i}\right)=\pi^{\mathrm{as}}\left(d, d_{-i}, z_{i}^{o}, \theta_{0}\right)+\nu_{2, i, d},
$$

and the distribution $\nu_{2, i, d}$ conditional on $\nu_{2,-i}$ has full support $[\forall(i, d)]{ }^{8}$

Keep in mind that the additive separability in equation (9) cannot be obtained definitionally. If we did observe realized profits and regressed it on $\left(d_{i}, d_{-i}, z_{i}^{o}\right)$, we would get a residual, but that residual is not the $\nu_{2, i}$ in equation (9). The regression residuals are mean independent of $\left(d_{i}, d_{-i}\right)$, while $\nu_{2, i}$

\footnotetext{
${ }^{8}$ Allowing for additional unobservables, for example, unobservable random coefficients on the $z_{i}^{o}$, would increase the notational burden but would not change our ability to obtain any of the results below.
} 
is not. So for the specification in equation (9) to be correct, $\pi^{\text {as }}(\cdot)$ and $\nu_{2, i}$ have to be derived from the primitives of the problem.

\section{Inequalities for Inference}

Index markets by $j=1, \ldots, J$. For inference, we need the distribution of $\left\{\left(\nu_{2, i, j}, z_{i, j}^{o}, d_{i, j}\right)\right\}_{i=1}^{n_{j}} \equiv\left(\nu_{2, j}, z_{j}^{o}, d_{j}\right)$ across the population of markets, which we denote by $P(\cdot)$. The data consist of random draws on $\left(\nu_{2, j}, z_{j}^{o}, d_{j}\right)$ from $P(\cdot)$. The expectations of any function $g(\cdot)$ of a draw conditional on $x$ will be given by $E[g(\cdot) \mid x]=\int g(\cdot) d P(\cdot \mid x)$, so $E(\cdot)$ is defined by the data generating process (DGP). The distribution of $\nu_{2, j}$ conditional on $z_{j}^{o}$ in DC4, or $F(\cdot ; \theta)$, is assumed to be consistent with this DGP.

The model's conditions can be satisfied by multiple vectors of $d_{j}$ for any value of $\theta$ (i.e., there can be multiple equilibria). As a result, there is not a one to one map between observables, unobservables, and parameters, on the one hand, and outcomes for the decision variables, on the other; so the model is not detailed enough to deliver a likelihood. However Ciliberto and Tamer (2009) and Andrews, Berry, and Jia (2006) noted that we can check whether the model's conditions are satisfied at the observed $d_{j}$ for any $\nu_{j}$ and $\theta$, and this, together with $F(\cdot ; \theta)$, enable us to calculate conditional probabilities of satisfying those conditions. Since these are necessary conditions for observing the choices made when $\theta=\theta_{0}$, the probability of satisfying them must be greater than the probability of actually observing $d_{j}$. In addition, if we checked whether the $d_{j}$ are the only values of the decision variables to satisfy the necessary conditions for each $\nu_{2, j}$ at that $\theta$, we could construct the probability that $d_{j}$ is the unique equilibrium. That probability must be lower than the true probability of observing $d_{j}$ at $\theta=\theta_{0}$. These are inequalities that not all values of $\theta$ will satisfy and as a result, they can be used as a basis for inference.

More formally define the probability that the model in equation (8) (with a restriction like that in equation (9)) is satisfied at a particular $d_{j}$ given $z_{j}^{o}$ for a given $\theta$ to be

$$
\overline{\operatorname{Pr}}\left\{d_{j} \mid z_{j}^{o}, \theta\right\} \equiv \operatorname{Pr}\left\{\nu_{2, j}: d_{j} \text { satisfy equation }(8) \mid z_{j}^{o}, \theta\right\}
$$

and the analogous lower bound to be

$$
\underline{\operatorname{Pr}}\left\{d_{j} \mid z_{j}^{o}, \theta\right\} \equiv \operatorname{Pr}\left\{\nu_{2, j} \text { : only } d_{j} \text { satisfy equation (8)| } z_{j}^{o}, \theta\right\} .
$$

Letting $I\{\cdot\}$ be the indicator function which takes the value 1 if the condition inside the brackets is satisfied and 0 elsewhere, the true probability (determined in part by the equilibrium selection mechanism) is

$$
\operatorname{Pr}\left\{d_{j} \mid z_{j}^{o}, \theta_{0}\right\} \equiv E\left[I\left\{d=d_{j}\right\} \mid z_{j}^{o}\right] .
$$

Since we do not know the selection mechanism, we do not know $\operatorname{Pr}\left\{d_{j} \mid z_{j}^{o}, \theta_{0}\right\}$, but we do know that when $\theta=\theta_{0}$,

$$
\overline{\operatorname{Pr}}\left\{d_{j} \mid z_{j}^{o}, \theta_{0}\right\} \geq \operatorname{Pr}\left\{d_{j} \mid z_{j}^{o}, \theta_{0}\right\} \geq \underline{\operatorname{Pr}}\left\{d_{j} \mid z_{j}^{o}, \theta_{0}\right\} .
$$


Let $h(\cdot)$ be a function which only takes on positive values and let $\rightarrow_{P}$ denote convergence in probability. Since $E(\cdot)$ provides cross-markets averages, we have

$$
\begin{aligned}
& E\left[J^{-1} \sum_{j}\left(\overline{\operatorname{Pr}}\left\{d_{j} \mid z_{j}^{o}, \theta\right\}-I\left\{d=d_{j}\right\}\right) h\left(z_{j}^{o}\right)\right] \\
& \quad \rightarrow_{P} J^{-1} \sum_{j}\left[\left(\overline{\operatorname{Pr}}\left\{d_{j} \mid z_{j}^{o}, \theta\right\}-\operatorname{Pr}\left\{d_{j} \mid z_{j}^{o}, \theta_{0}\right\}\right) h\left(z_{j}^{o}\right)\right],
\end{aligned}
$$

which is nonnegative at $\theta=\theta_{0}$. An analogous moment condition can be constructed from $\operatorname{Pr}\left\{d_{j} \mid z_{j}^{o}, \theta_{0}\right\}-\underline{\operatorname{Pr}}\left\{d_{j} \mid z_{j}^{o}, \theta\right\}$. The estimation routine constructs unbiased estimates of $(\underline{\operatorname{Pr}}(\cdot \mid \theta), \overline{\operatorname{Pr}}(\cdot \mid \theta))$, substitutes them for the true values of the probability bounds into these moments, and then accepts values of $\theta$ for which the moment inequalities are satisfied. ${ }^{9}$

Since typically neither the upper nor the lower bound is an analytic function of $\theta$, simulation is used to obtain unbiased estimates of them. The simulation procedure is straightforward, though often computationally burdensome. Take pseudorandom draws from a standardized version of $F(\cdot)$ as defined in DC4 and for each random draw, check the necessary conditions for an equilibrium, that is, the conditions in equation (8), at the observed $\left(d_{i}, d_{-i}\right)$. Estimate $\overline{\operatorname{Pr}}\left(d_{i}, d_{-i} \mid \theta\right)$ by the fraction of random draws that satisfy those conditions at that $\theta$. Next check if there is another value of $\left(d, d_{-i}\right) \in D_{i} \times D_{-i}$ that satisfies the equilibrium conditions at that $\theta$ and estimate $\underline{\operatorname{Pr}}\left(d_{i}, d_{-i} \mid \theta\right)$ by the fraction of the draws for which $\left(d_{i}, d_{-i}\right)$ is the only such value. If we were analyzing markets with $N$ interactive agents, each of whom had \#D possible choices and used $n s$ simulation draws on $\left\{\nu_{2, i}\right\}_{i=1}^{N}$, then for each market and each $\theta$ evaluated in the estimation routine, we need to evaluate up to $n s \times \# D \times N$ inequalities to obtain estimates of $\overline{\operatorname{Pr}}\{\cdot \mid \theta\}$, and we need to evaluate up to $n s \times(\# D)^{N}$ inequalities if we also estimate $\underline{\operatorname{Pr}}(\cdot \mid \theta)$. This can be computationally expensive, particularly in multistage games solved by backward recursions, as then to solve for each $\pi\left(d_{i}, d_{-i}, \cdot\right)$ we need to compute equilibria to later stages of the game.

\subsection{Profit Inequalities}

An earlier version of this approach appears in Pakes, Porter, Ho, and Ishii (2006). Recall that what we need is an assumption on the relationship between

\footnotetext{
${ }^{9}$ As noted by a referee, this routine ignores information. If we can enumerate all possible equilibria, as is assumed if we use the lower bound, we could use the fact that the equilibrium selection probabilities must sum to 1 (for more detail, see Beresteanu and Molinari (2008)). Also, I have implicitly assumed that there is an equilibrium in pure strategies for each point evaluated. This need not be the case; for a discussion of the implications of existence problems for econometric work on discrete games, see Bajari et al. (2010).
} 
(i) the agents' perceptions of expected returns and the returns emanating from the data generating process and (ii) the profit measure the agent uses and the one the econometrician specifies.

From equation (5), $\mathcal{E}\left[\Delta \pi\left(\cdot, \theta_{0}\right) \mid \mathcal{J}_{i}\right] \geq 0$. If $x_{i} \in \mathcal{J}_{i}$ this implies $\mathcal{E}\left[\Delta \pi\left(\cdot, \theta_{0}\right) \mid\right.$ $\left.x_{i}\right]=0$. PC 3 relates these expectations to averages from the data generating process (our $E(\cdot)$ operator).

PC3: There is a positive valued $h(\cdot)$ and an $x_{i} \in J_{i}$ for which

$$
\begin{aligned}
& \frac{1}{N} \sum_{i} \mathcal{E}\left(\Delta \pi\left(d_{i}, d^{\prime}, \mathbf{d}_{-i}, \mathbf{z}_{i}, \theta_{0}\right) \mid x_{i}\right) \geq 0 \\
& \quad \Rightarrow \quad E\left(\frac{1}{N} \sum_{i} \Delta \pi\left(d_{i}, d^{\prime}, \mathbf{d}_{-i}, \mathbf{z}_{i}, \theta_{0}\right) h\left(x_{i}\right)\right) \geq 0 .
\end{aligned}
$$

PC3 nests DC3, as it allows for uncertainty and it does so without requiring us to fully specify how the agent forms his expectations. If agents know (i) the other agents' strategies (i.e., $\mathbf{d}_{-i}\left(\mathcal{J}_{-i}\right)$ ), and (ii) the joint distribution of other agents' information sets and the primitives sources of uncertainty (i.e., of $\left(\mathcal{J}_{-i}, \mathbf{z}_{i}\right)$ conditional on $\left.\mathcal{J}_{i}\right)$, then, provided all expectations exist, our optimality condition $\mathrm{C} 1$ insures that $\mathrm{PC} 3$ is satisfied. These assumptions are, however, stronger than are needed for PC3. Several authors have noted that agents' expectations can satisfy $\mathrm{C} 1$ without them having such detailed information (see, e.g., Dekel, Fudenberg, and Levine (1993)). Furthermore, although correct expectations about profit differences are sufficient for PC3, they are not necessary. A weaker sufficient condition is

$$
\begin{aligned}
& \frac{1}{N} \sum_{i} \mathcal{E} \Delta \pi\left(d_{i}, d^{\prime}, \mathbf{d}_{-i}, \mathbf{z}_{i}, \theta_{0}\right) h\left(x_{i}\right) \\
& \quad-E\left(\frac{1}{N} \sum_{i} \Delta \pi\left(d_{i}, d^{\prime}, \mathbf{d}_{-i}, \mathbf{z}_{i}, \theta_{0}\right) h\left(x_{i}\right)\right) \geq 0,
\end{aligned}
$$

where again $E(\cdot)$ is defined by the DGP. If agents have incorrect expectations on $\Delta \pi\left(\cdot, \theta_{0}\right)$ but their expectational error is not systematically related to $x_{i}$ (i.e., are mean independent of $x_{i}$ ), then (11) is satisfied with equality. Indeed PC3 is satisfied even if agents are incorrect on average, provided they are overly optimistic about the relative profitability of their choices.

The final condition used in this estimation strategy is designed to deal with the selection problem caused by the $\left\{\nu_{2, i, j, d}\right\}$ for the $i=1, \ldots, N_{j}$ agents in market $j$. C1, C2, and the definitions in equation (7) imply that our model generates the restriction that

$$
\mathcal{E}\left[\Delta \pi\left(\cdot ; \theta_{0}\right) \mid \mathcal{J}\right]=\mathcal{E}\left[\Delta r\left(\cdot ; \theta_{0}\right) \mid \mathcal{J}\right]-\Delta \nu_{2} \geq 0 .
$$


PC3 insures that this implies that if $x \in \mathcal{J}$, sample averages of $\Delta \pi\left(\cdot ; \theta_{0}\right) h(x)=$ $\Delta r\left(\cdot ; \theta_{0}\right) h(x)-\Delta \nu_{2} h(x)$ have positive expectation. For consistency we require that the sample average of the observable $\Delta r(\cdot ; \theta) h(x)$ have positive expectation at $\theta=\theta_{0}$. This will be the case if the expectation of the average of $\nu_{2} h(x)$ is nonnegative. Above we stressed that even if $x$ is an instrument in the sense that $\left\{\nu_{2, d}\right\}$ is mean independent of $x$ in the population at large, the mean of $\nu_{2, d}$ conditional on $x$ for those who made particular decisions will typically depend on $x$. Pakes et al. (2006) presented a general condition which insures that the selection resulting from conditioning on agents' choices does not impact the consistency of our estimators. Here we consider three ways to form moments that satisfy that condition that we have seen used, often in combination, in applied work. They are based on the researcher finding weighted averages of differences between actual and counterfactual choices that either (i) difference out the effect of the $\nu_{2}$ (PC4a), (ii) insure that we average over the $\nu_{2}$ of every agent (so that there is no selection) (PC4b), or (iii) sum to an observable which controls for a weighted average of the $\nu_{2}(\mathrm{PC} 4 \mathrm{c})$.

PC4a-Differencing: Let there be $G$ groups of observations indexed by $g$, counterfactuals $d_{i, g}^{\prime} \in \mathcal{D}_{i, g}$, and positive weights $w_{i, g} \in \mathcal{J}_{i, g}$ such that $\sum_{i \in g} w_{i, g} \times$ $\Delta \nu_{2, i, g, d_{i, g}, d_{i, g}^{\prime}}=0$; that is, a within-group weighted average of profit differences eliminates the $\nu_{2}$ errors. Then

$$
G^{-1} \sum_{g} \sum_{i \in g} w_{i, g}\left(\Delta r\left(d_{i, g}, d_{i, g}^{\prime}, \cdot ; \theta_{0}\right)-\mathcal{E}\left[\Delta \pi\left(d_{i, g}, d_{i, g}^{\prime}, \cdot ; \theta_{0}\right) \mid \mathcal{J}_{i, g}\right]\right) \rightarrow_{P} 0,
$$

provided $G^{-1} \sum_{g} \sum_{i} w_{i, g} \Delta r\left(d_{i, g}, d_{i, g}^{\prime}, \cdot ; \theta_{0}\right)$ obeys a law of large numbers.

Our Case 1 supermarket example is a special case of PC4a with $n_{g}=w_{i, g}=$ 1: There $d_{i}=\left(b_{i}, s_{i}\right), \pi(\cdot)=U\left(b_{i}, z_{i}\right)-e\left(b_{i}, s_{i}\right)-\theta_{0} d t\left(s_{i}, z_{i}\right)$, and $\nu_{2, i, d} \equiv$ $U\left(b_{i}, z_{i}\right)$. If we measure expenditures up to a $\nu_{1, i, d}$ error, $r(\cdot)=-e\left(b_{i}, s_{i}\right)-$ $\theta_{0} d t\left(s_{i}, z_{i}\right)+\nu_{2, i, d}+\nu_{1, i, d}$. We chose a counterfactual with $b_{i}^{\prime}=b_{i}$, so $\Delta r(\cdot)=$ $\Delta \pi(\cdot)+\Delta \nu_{1}$, and the utility from the bundle of goods bought is differenced out. "Matching estimators," that is, estimators based on differences in outcomes of matched observations, implicitly assume PC4a (no differences in unobservable determinants of the choices made by matched observations). For other single agent examples, see Pakes et al. (2006).

For a multiple agent example, consider two period entry games with common unobservable determinants of market profitability-the problem that stimulated the literature on using inequality estimators in multiple agent settings. For specificity, consider two retailers, say $i=\{W, T\}$, deciding whether to enter different markets, so $d_{j}^{i} \in\{1,0\}$ and $d_{j}^{i}=1$ indicates that firm $i$ enters market $j=[1, \ldots, J]$. If there are market-specific unobservables 
known to the agents but not to the econometrician, then $r_{j}\left(d_{i, j}, d_{-i, j}, z_{i}, \theta\right)=$ $\mathcal{E}\left[\pi_{j}\left(d_{i, j}, \mathbf{d}_{-i, j}, \mathbf{z}_{i}, \theta\right) \mid J_{i}\right]+\nu_{2, j}+\nu_{1, i, j}$. The $z_{i}$ include sources of cost differences (like warehouse and central office locations). Let $w_{i, j}=w_{j} \in\left\{0, J^{-1}\right\}$ with $w_{j}=J^{-1} \Leftrightarrow d_{j}^{T}=\left[1-d_{j}^{W}\right]$; that is, $\sum_{j} \sum_{i} w_{i, j} \Delta \pi_{i, j}(\cdot)$ puts weight only on markets where the two agents make opposite decisions.

The only possible counterfactual is $d_{j}^{i, \prime}=\left[1-d_{j}^{i}\right]$. So if $w_{j}=1$ and $d_{j}^{W}=1$, then $d_{j}^{T}=0, \Delta r_{j}^{W}(\cdot)=\mathcal{E}\left[\Delta \pi_{j}^{W}(\cdot) \mid \mathcal{J}_{W}\right]+\nu_{2, j}+\nu_{1, W, j}$, and $\Delta r_{j}^{T}(\cdot)=$ $\mathcal{E}\left[\Delta \pi_{j}^{T}(\cdot) \mid \mathcal{J}_{T}\right]-\nu_{2, j}+\nu_{1, T, j}$. Since $\nu_{2, j}$ enters the two inequalities with opposite signs, it cancels when we sum over $i=\{W, T\}$ and

$$
\begin{aligned}
& \sum_{j} w_{j} \sum_{i=W, T} \Delta r_{j}^{i}\left(d^{i}, d^{i, \prime}, \mathbf{d}_{-i}, \mathbf{z}_{i}, \theta\right) \\
& \quad=\sum_{j} w_{j} \sum_{i=W, T}\left(\mathcal{E}\left[\Delta \pi_{j}^{i}\left(d^{i}, d^{i, \prime}, \mathbf{d}_{-i}, \mathbf{z}_{i}, \theta\right) \mid \mathcal{J}_{i}\right]+\nu_{1, i, j}\right),
\end{aligned}
$$

which will be nonnegative at $\theta=\theta_{0}$.

Notice that since we assume $w_{i, j} \in \mathcal{J}_{i, j}$, these weights imply $d_{-i, j} \in \mathcal{J}_{i, j}$, as in the generalized discrete choice model, but we do not require assumptions on the distribution of $\mathbf{z}_{i}$ or of $\nu_{i, .}$ Also more moment inequalities can be generated from appropriate instruments and the model can be enriched to explicitly allow for differences in the firm's responses to the market-specific shock (replace $\nu_{2, j}$ by $\theta z_{j}^{i} \nu_{2, j}$, where $z_{j}^{i} \geq 0$ and $z_{j}^{i} \in J_{i}$, and then divide each difference by $z_{j}^{i}$ ). Similar structures appear in a number of other familiar problems (e.g., social interaction models where the interaction effects are additive and groupspecific).

In simultaneous move games where the market allocation mechanism is known, one can often construct counterfactuals which difference out individ$u a l$-specific (rather than group-specific) $\nu_{2}$ effects. For example, in electricity auctions with known allocation mechanisms, we can compute the difference between the revenues and quantities actually allocated to the agent, and those the agent would have obtained had the agent submitted a different bid (holding the realizations of environmental variables and competitors' bids constant). Profits are revenues minus fixed and variable costs. The fact that the expectation of the difference in profits from the two bids should be positive allows us to bound the variable cost function without restricting agent-specific fixed costs in any way (as they are differenced out).

PC4b-Unconditional Averages and Instrumental Variables: Assume that $\forall d \in D_{i}$, there is a $d^{\prime} \in D_{i}$ and a $w_{i} \in \mathcal{J}_{i}$ such that

$$
w_{i} \Delta r\left(d_{i}, d_{i}^{\prime}, \cdot ; \theta\right)=w_{i} \mathcal{E}\left[\Delta \pi\left(d_{i}, d_{i}^{\prime}, \cdot ; \theta\right) \mid \mathcal{J}_{i}\right]+\nu_{2, i}+\Delta \nu_{1, i, \cdot} .
$$


Then if $x_{i} \in \mathcal{J}_{i}, E\left[\nu_{2, i} \mid x_{i}\right]=0$, and $h(\cdot)>0$,

$$
\begin{aligned}
& N^{-1} \sum_{i} w_{i} \Delta r\left(d_{i}, d_{i}^{\prime}, \cdot ; \theta_{0}\right) h\left(x_{i}\right) \\
& \quad \rightarrow_{P} N^{-1} \sum_{i} w_{i} \mathcal{E}\left[\Delta \pi\left(d_{i}, d_{i}^{\prime}, \cdot ; \theta_{0}\right) \mid \mathcal{J}_{i}\right] h\left(x_{i}\right) \geq 0,
\end{aligned}
$$

provided $N^{-1} \sum \nu_{1, i}, h\left(x_{i}\right)$ and $N^{-1} \sum \nu_{2, i} h\left(x_{i}\right)$ obey laws of large numbers.

$\mathrm{PC} 4 \mathrm{~b}$ assumes there is a counterfactual which gives us an inequality that is additive in $\nu_{2}$ no matter what decision the agent made. Then we can form averages which do not condition on $d$, and hence do not have a selection problem. This form of PC4b suffices for the examples in this section but the next section requires a more general form, which allows us to group agents and is given in the footnote below. ${ }^{10}$

Case 2 of our supermarket example had two $\nu_{2}$ components: a decisionspecific utility from the goods bought, $\nu_{2, i, d}=U\left(b_{i}, z_{i}\right)$ (as in Case 1), and an agent-specific aversion to drive time, $\theta_{i}=\theta_{0}+\nu_{2, i}$. As in Case 1, taking $d^{\prime}=\left(b_{i}, s_{i}^{\prime}\right)$ differenced out the $U\left(b_{i}, z_{i}\right)$. Then $\Delta r(\cdot)=-\Delta e\left(\cdot, s_{i}, s_{i}^{\prime}\right)-\left(\theta_{0}+\right.$ $\left.\nu_{2, i}\right) \Delta d t\left(s_{i}, s_{i}^{\prime}, z_{i}\right)+\Delta \nu_{1, .}$. Divide by $\Delta d t\left(s_{i}, s_{i}^{\prime}, z_{i}\right) \leq 0$. Then C1 and C2 imply that $\mathcal{E}\left[\Delta e\left(s_{i}, s_{i}^{\prime}, b_{i}\right) / \Delta d t\left(s_{i}, s_{i}^{\prime}, z_{i}\right) \mid \mathcal{J}_{i}\right]-\left(\theta_{0}+\nu_{2, i}\right) \leq 0$. This inequality is (i) linear in $\nu_{2, i}$ and (ii) is available for every agent. So if $E\left[\nu_{2}\right]=0$, PC3 and a law of large numbers insure $N^{-1} \sum_{i} \nu_{2, i} \rightarrow_{P} 0$, and $\sum_{i} \Delta e\left(s_{i}, s_{i}^{\prime}, b_{i}\right) / \Delta d t\left(s_{i}, s_{i}^{\prime}, z_{i}\right) \rightarrow_{P}$ $\underline{\theta}_{0} \leq \theta_{0}$ : whereas if $E\left[\nu_{2} \mid x\right]=0$, we can use $x$ to form instruments. Notice that $\nu_{2, i}$ can be correlated with $d t\left(z_{i}, s_{i}\right)$, so this procedure enables us to analyze discrete choice models when a random coefficient affecting tastes for a characteristic is correlated with the characteristics chosen.

For a multiple agent example of PC4b, we look at within market expansion decisions. Agents chose a number of outlets, a $d_{i} \in \mathcal{Z}_{+}$(the integers) to maximize expected profits. Formally the model is a multiple agent two period ordered choice model: a model with many Industrial Organization applications (e.g., Ishii (2008)). In the first period the agents chose a number of outlets and in the second they obtain the variable profits from sales at those outlets. So $\pi_{i}(\cdot)=v p\left(d_{i}, d_{-i}, \cdot\right)-\left(c_{0}+\nu_{2, i}\right) d_{i}$, where $v p(\cdot)$ are variable profits and $\left(c_{0}+\nu_{2, i}\right)$ represent the costs of building and maintaining the outlets. These costs differ across firms in ways known to the firms but not to the econometrician, and $c_{0}$ is defined to be their average (so $\sum \nu_{2, i} \equiv 0$ ). We measure variable profits up to a $\nu_{1}(\cdot)$ measurement error, so define $r_{i}(\cdot)=\pi_{i}(\cdot)+\nu_{1, d_{i}}=$ $v p_{i}(\cdot)-c_{o} d_{i}+\nu_{2, i} d_{i}+\nu_{1, d_{i}}$, where $v p_{i}(\cdot)$ and $d_{i}$ are observed.

\footnotetext{
${ }^{10}$ The more general version is as follows. Assume $G$ groups of observations with $n_{g}$ members in group $g$, and that for each $\left\{d_{i, g} \in D_{i, g}\right\}_{i}$ there is a counterfactual $\left\{d_{i, g}^{\prime} \in D_{i, g}\right\}_{i}$ and positive weights $w_{i, g} \in \mathcal{J}_{i, g}$ such that $\sum_{i \in g} w_{i, g} \Delta r\left(d_{i, g}, d_{i, g}^{\prime}, \cdot, \theta_{0}\right)=\sum_{i \in g} w_{i, g}\left(\mathcal{E}\left[\Delta \pi\left(d_{i, g}, d_{i, g}^{\prime}, \cdot, \theta_{0}\right) \mid \mathcal{J}_{i, g}\right]+\right.$ $\left.\nu_{2, g}+\nu_{1, i, g}\right)$. Then if $x_{i, g} \in \mathcal{J}_{i, g}, E\left[\nu_{2, g} \mid x_{i, g}\right]=0$, and $h(\cdot)>0, G^{-1} \sum_{g} \sum_{i \in g} h\left(x_{i, g}\right)\left(w_{i, g} \Delta r\left(d_{i, g}\right.\right.$, $\left.d_{i, g}^{\prime}, \cdot ; \theta_{0}\right) \rightarrow_{P} G^{-1} \sum_{g} \sum_{i \in g} h\left(x_{i, g}\right)\left(w_{i, g} \mathcal{E}\left[\Delta \pi\left(d_{i, g}, d_{i, g}^{\prime}, \cdot ; \theta_{0}\right) \mid \mathcal{J}_{i, g}\right] \geq 0\right.$, provided laws of large numbers hold.
} 
$\mathrm{C} 1$ and $\mathrm{C} 2$ imply that the incremental profits from choosing one more machine than was actually chosen $\left(\right.$ a $\left.d_{i}^{\prime}=d_{i}+1\right)$ are expected to be less than its cost, or $\mathcal{E}\left[\Delta \pi\left(d_{i}, d_{i}+1, \cdot\right) \mid \mathcal{J}_{i}\right] \leq 0$. But $\Delta r\left(d_{i}, d_{i}+1, \cdot\right)=\mathcal{E}\left[\Delta \pi\left(d_{i}, d_{i}+\right.\right.$ $\left.1, \cdot) \mid \mathcal{J}_{i}\right]+\nu_{2, i}+\Delta \nu_{1, i, .}$. So since $\sum \nu_{2, i} \equiv 0$,

$$
\begin{aligned}
N^{-1} \sum_{i} \Delta r\left(d_{i}, d_{i}+1, \cdot\right) & \rightarrow_{P} N^{-1} \sum_{i} \Delta \pi\left(d_{i}, d_{i}+1, \cdot\right) \\
& =N^{-1} \sum_{i}\left[\Delta v p\left(d_{i}, d_{i}+1, \cdot\right)-c_{0}\right] \leq 0,
\end{aligned}
$$

where we have assumed PC3 and a law of large numbers. That is, $N^{-1} \times$ $\sum_{i}\left[\Delta r\left(d_{i}, d_{i}+1, \cdot\right) \rightarrow_{P} \underline{c}_{0} \leq c_{0}\right.$. An upper bound for $c_{0}$ can be obtained by choosing $d_{i}^{\prime} \leq d_{i}$ (see Pakes et al. (2006), for the case where some observations are at $d_{i}=0$, in which case the counterfactual $d_{i}^{\prime}<d_{i}$ is infeasible). Additional moments can be obtained by forming covariances with $h\left(x_{i}\right)$ that are (unconditionally) uncorrelated with $\nu_{2, i}$. Notice that $E\left[v_{2} h(x)\right]=0$ is our only assumption; in particular we do not require $d_{-i} \in \mathcal{J}_{i}$ as in our previous multiple agent example.

PC4c-Control Functions: Often variables that are not available at a disaggregated level are available as aggregates, and this can be used to develop a control function for $\nu_{2}$. Two familiar examples are (i) firm level exports to different countries are typically unavailable but aggregate trade flows by product and country of destination are recorded, and (ii) product level input, cost, and sales data of multiproduct firms are not typically available, but both firm level aggregates over products, and product level aggregates over firms, often are. We illustrate with a firm location application adapted from de Loecker et al. (2010) that uses the trade data. An alternative illustration would have been to use the available data on multiproduct firms in conjunction with the product level aggregates over firms to analyze multiproduct cost functions.

The output of each firm at each location is known, but where that output is sold is not. The fixed costs of firm $i$ in location $d$ are $f\left(z_{i}, d, \theta\right)$, while its marginal costs are $m\left(z_{i}, d ; \theta\right)$ (both vary with location of production and firm characteristics). Let $q_{i, d}$ be the quantity firm $i$ produces and let $q_{i, e}=q_{e}+$ $\nu_{2, i}^{e}$ be the quantity it exports to market $e$. Here $q_{e}$ is average exports to $e$, so $\sum_{i} \nu_{2, i}^{e} \equiv 0(\forall e) . c(d, e)$ is the transportation costs (which vary with the location of production $d$ and consumption $e$ ). We observe $q_{i, d}$ and $z_{i}$, and measure the firm's total cost up to a $\nu_{1}$ error, or $r(\cdot)$, where

$$
\begin{aligned}
r\left(z_{i}, d ; \theta\right)= & f\left(z_{i}, d ; \theta\right)+m\left(z_{i}, d ; \theta\right) q_{i, d} \\
& +\sum_{e} c(d, e, \theta) q_{e}+\sum_{e} c(d, e, \theta) \nu_{2, i}^{e}+\nu_{1, i, d} .
\end{aligned}
$$


Each firm producing in $d$ chose its location to minimize expected costs and each could have produced in counterfactual location $d^{\prime}$ without changing the countries it sold to. Summing the expected cost difference in moving all firms from $d$ to $d^{\prime}$, letting $Q^{e}$ be (the observed) total exports to $e$, and using $\sum_{i} \sum_{e} c(d, e) \nu_{2, i}^{e}=0$, we have

$$
\begin{aligned}
& N^{-1} \sum_{i} \Delta r\left(d, d^{\prime}, \cdot, \theta\right) \\
& \quad \rightarrow_{P} N^{-1} \sum_{i}\left(\Delta f\left(d, d^{\prime}, \cdot, \theta\right)+\Delta m\left(d, d^{\prime}, \cdot, \theta\right) q_{i, d}\right) \\
& \quad+\sum_{e} \Delta c\left(d, d^{\prime}, e, \theta\right) Q^{e} \leq 0
\end{aligned}
$$

at $\theta=\theta_{0}$, which allows us to combine the microdata on firms costs and aggregate data on exports to bound the parameters of interest. Notice that we do so without having to either estimate demand functions or make a pricing assumption in each country.

\subsection{Comparing the Two Approaches}

The two approaches differ in their informational (DC3 vs. PC3) and their measurement (DC4 vs. PC4) assumptions. They also differ in their computational properties, but these are discussed in the context of the Monte Carlo example in Section 4.

PC3 nests DC3. PC3 allows for uncertainty and does so without having to specify either the agents' information sets or their subjective probability distributions conditional on those information sets, objects we typically know little about. It also allows for expectational errors provided they are mean independent of the instruments. DC3 assumes agents know the returns from every choice and correctly optimize. Partly as a result, DC3's primary use in multiple agent settings has been as a characterization of "rest points" of environments which are "stable" over time- a setting often invoked to justify the use of two period games to structure cross sectional empirical work. Notice that even in this setting, the combination of DC3 with DC4 only leads to consistent bounds if the profit (or value) function is constructed from correctly specified primitives. If instead a reduced form from regressing profits on variables of interest is used, the model contain a regression error. This violates the assumptions of the generalized discrete choice model and will lead to inconsistent estimates of the bounds on the reduced form parameters. We show how to adapt the discrete choice model to accommodate reduced forms below.

The two measurement assumptions (PC4 and DC4) are not nested. Indeed the simplest of the measurement assumptions used to justify the two models are distinctly different. Sufficient conditions for PC4 are that the $\left\{\nu_{2, i, d}\right\}$ do not vary over $d$ and that there are instruments which are uncorrelated with the 
$\left\{\nu_{1, i, d}\right\}$. DC4 requires that the $\left\{\nu_{1, i, d}\right\}$ do not vary over $d$ and that there be a known joint distribution for the $\left\{\nu_{2, i, d}\right\}$. When $\left\{\nu_{2, i, d}\right\}$ does vary over $d$, then to use the profit inequalities approach, we need to control for a selection problem. Our ability to do so typically depends on the richness of the set of feasible counterfactual choices and the appropriate form(s) for the heterogeneity. When $\left\{\nu_{1, i, d}\right\}$ does vary over $d$, then the generalized discrete choice model will not deliver consistent bounds. $\left\{\nu_{1, i, d}\right\}$ will vary over $d$ if there is uncertainty about outcomes, measurement error, or specification error. For some of these cases, we can modify the generalized discrete choice model and its estimation algorithm to deliver consistent bounds.

For a familiar example, consider the case where we are analyzing the profits from entering markets in which the true profit from entering is additively separable in $\nu_{2}$, or $\pi\left(d_{i, j}=1, d_{-i, j}, z_{i, j}^{o}, \nu_{2, i, j}\right)=\pi^{\text {as }}\left(d_{i, j}=1, d_{-i, j}, z_{i, j}^{o}, \theta_{0}\right)+\nu_{2, i, j}$, as in equation (9) and the profits from not entering are normalized to zero. As in most entry models, we are interested in the reduced form obtained by regressing $\pi^{\text {as }}(\cdot)$ onto $z_{i, j}^{o}$ and the number of competitors, say $\sum_{i}\left\{d_{i, j}=1\right\}$. So

$$
r\left(d_{i, j}=1, d_{-i, j}, z_{i, j}^{o}, \theta\right)=z_{i, j}^{o} \theta_{z}+\sum_{i}\left\{d_{i, j}=1\right\} \theta_{d}+\nu_{2, i, j}+\nu_{1, i, j},
$$

where $\nu_{1, i, j}$ is the regression error, so $E\left[\nu_{1, i, j} \mid z_{i, j}^{o}, \sum_{i}\left\{d_{i}=1\right\}, \nu_{2, i, j}\right]=0$ by construction. Recall that to obtain the inequalities used in estimation in this model, we have to check whether the Nash conditions are satisfied at a given $\theta$; that is, to check whether $d_{i, j}$ maximizes $z_{i, j}^{o} \theta_{z}+\sum\left\{d_{i, j}=1\right\} \theta_{d}+\nu_{2, i, j}+\nu_{1, i, j}$ conditional on $d_{-i, j}$ for each $i$. To do this, we will need an assumption on the joint distribution of the $\nu_{1, i, j}$, as well as for the $\left\{\nu_{2, i, j}\right\}$; and the $\nu_{1, i, j}$ must be mean independent of the $\left\{\nu_{2, i, j}\right\}, d_{i, j}$, and $z_{i, j}$, while the $\left\{\nu_{2, i, j}\right\}$ are determinants of $d_{i, j}$. Given the additional distributional assumption the estimation algorithm is analogous to that in Section 2.3, although a more complex simulator must be used.

It is not as easy to accommodate measurement error in the generalized discrete choice framework. If $z_{i, j}^{o}=z_{i, j}^{*}+\nu_{1, i, j}$ and the agent responds to $z^{*}$ (not $z^{o}$ ), estimation requires checking whether $z_{i, j}^{*} \theta_{z}+\sum_{i}\left\{d_{i, j}=1\right\} \theta_{d}+\nu_{2, i, j, d}$ satisfies the Nash conditions. To obtain a simulator that would allow us to do so, we would need to draw from the distribution of $z^{*}$ conditional on $z^{0}$. This is typically not known nor is it easy to estimate. In contrast, classical measurement error does not affect the consistency of the profit inequality estimator.

Finally DC4 requires explicit distributional assumptions on the $\left\{\nu_{2, i, j}\right\}$, while the profit inequality model relies only on mean independence assumptions. The need for an explicit distributional assumption is a concern, as the generalized discrete choice corrects for selection by finding the probability of $\nu_{2}$ values which induce the agent to make particular choices. Those probabilities depend on the properties of the tail of the $\nu_{2}$ distributions-properties we often know little about. The Monte Carlo example in Section 4 is designed to investigate 
the robustness of the two models to violations of their assumptions and indicates that it is the distributional assumption of the generalized discrete choice model that is most problematic.

\section{A MULTIPLE AGENT EXAMPLE (BUYER-SELLER NETWORKS)}

Vertical markets typically contain a small number of both sellers and buyers (who resell the products they buy to consumers). Most buyers buy from more than one seller, while most sellers sell to more than one buyer. The terms of the payments the buyer makes to the seller are negotiated and vary with underlying market conditions. These terms determine both the costs buyers factor into the prices they set when they remarket the goods they sell to consumers and the split of the profits between the sellers and the buyers, and hence the sellers' incentives to invest in cost reductions (or product improvements). Unfortunately those terms are often proprietary; a seller bargaining with many buyers may not want one buyer to know the terms of its other contracts.

Costs are also often proprietary in consumer goods markets. However, since these are markets with many purchasers, we typically assume sellers have the power to set prices (or quantities) in them. Then the first order conditions from a Nash equilibrium can be used to back out costs; that is, we can find the marginal costs that insure that no firm has an incentive to deviate from the observed prices. This section uses moment inequalities to unravel features of the payment structure in vertical markets in an analogous way. We observe which sellers establish contracts with which buyers and, were we to know the buyer's cost function, could compute approximations to both the buyers and the sellers profits from (i) the existing arrangement and from (ii) a counterfactual in which one of the observed relationships is changed. So we proceed by parameterizing the buyer's cost function and look for values of the parameter vector that, on average, make the profits from the observed contracts larger than those from possible counterfactuals; that is, values of $\theta$ that make the observed relationship in the interests of both agents.

We analyze an HMO-hospital example. To see how market characteristics effect payments in this example, consider two different situations. In one a hospital with excess capacity in a neighborhood with several other similar noncapacity constrained hospitals is bargaining with an HMO. The HMO has already contracted with other neighborhood hospitals. Since there are similar options for consumers who require a hospital, the HMO's attractiveness to consumers is relatively insensitive to the inclusion of the given hospital in its network. As a result, were the HMO to include that hospital it would not, in equilibrium, increase the premium it charges to consumers. So for the hospital's contract offer to be accepted by the HMO, the contract would have to set hospital prices low enough for the HMO to prefer sending patients to that particular hospital rather than to its neighbors. On the other hand, if the hospital was the only hospital in the neighborhood, the HMO would be unlikely to 
attract any customers from that neighborhood without having the hospital in its network. Then, provided it is in the HMO's interest to operate in the neighborhood, the hospital should be able to extract nearly all the (hospital related) premiums that would be generated in that neighborhood.

To use this logic in estimation, we will have to specify what the buyer (seller) would have expected to happen if he had made a counterfactual choice. This requires assumptions, although the assumptions need not specify the form of the contracts. We suffice with a reduced form for the payments generated by the contracts obtained by regressing the HMO's per patient payments on hospital characteristics. In these respects, this paper follows the assumptions used in Ho's (2009) work on HMO-hospital markets. Ho's analysis assumes that there are no structural disturbances in her data (in our notation, $\nu_{2, i, d}=\nu_{2, i}$ $\forall d)$. We begin by showing that, by changing the moment inequalities taken to data, we can develop an estimation algorithm for her model that allows for both $\nu_{2}$ and $\nu_{1}$ errors. We then compare the results from estimators that do and that do not allow for $\nu_{2}$ errors. Next full information equilibria are computed from a structural buyer-seller network game with primitives similar to those in the empirical example. The reduced form implied by the computed equilibria is calculated, compared to the estimates obtained from the inequality estimators, and explored for possible additional correlates of contract characteristics.

\subsection{Empirical Analysis}

Ho (2009) used a two period game to structure the analysis: in the first period, contracts between HMOs and hospitals are established, and in the second period, the HMOs engage in a premium setting game which conditions on those contracts (and is assumed to have a unique Nash equilibria). Once the premiums are set, consumers choose HMO's and, if the need arises, choose a hospital in their HMO's network.

The premium setting game generates revenues for each HMO conditional on any configuration of hospital networks. Let $H_{m}$ be a vector of dimension equal to the number of hospitals whose components are either 0 or 1 , where a 1 indicates the hospital is in HMO $m$ 's network and a 0 indicates it is not. $H_{-m}$ specifies the networks of the competing HMOs. The revenues the HMO receives from the premium setting game, say $R_{m}\left(H_{m}, H_{-m}, z\right)$, and the number of patients HMO $m$ sends to hospital $h$, say $q_{m, h}\left(H_{m}, H_{-m}, z\right)$, depend on these networks and exogenous variables (our $z$ ).

The profits of the HMO are the revenues from the second period game minus the transfers the HMO makes to the hospitals in its network in payment for their services, say $T_{m, h}$ or

$$
\pi_{m}^{M}\left(H_{m}, H_{-m}, z\right)=R_{m}\left(H_{m}, H_{-m}, z\right)-\sum_{h \in H_{m}} T_{m, h}\left(H_{m}, H_{-m}, z\right) .
$$


Analogously if $c_{h}$ is the per patient costs of hospital $h$ and $M_{h}$ is the hospital's network of HMOs, the hospital's profits are

$$
\begin{aligned}
\pi_{h}^{H}\left(M_{h}, M_{-h}, z\right)= & \sum_{m \in M_{h}} T_{m, h}\left(H_{m}, H_{-m}, z\right) \\
& -c_{h} \sum_{m \in M_{h}} q_{m, h}\left(H_{m}, H_{-m}, z\right) .
\end{aligned}
$$

We project $T_{m, h}$ onto a set of interactions of $q_{m, h}(\cdot)$ with a vector of hospital characteristics, say $z_{h}$, and look for bounds on the resulting reduced form parameters; that is, if $x_{m, h}(\cdot)=q_{m, h}(\cdot) z_{h}$ are the interactions, we estimate the $\theta$ in $T_{m, h}\left(H_{m}, H_{-m}, z\right)=x_{m, h}\left(H_{m}, H_{-m}, z\right) \theta+\nu_{m, h}$, where $\nu_{m, h}$ are uncorrelated with $x_{m, h}$ by construction. Note that if agents know more about the details of the contracts they sign than is captured by $x_{m, h}(\cdot), \nu_{m, h}$ has a component which is known to both agents when they make their decisions (a " $\nu_{2}$ " component). Substituting this form of $T_{m, h}(\cdot)$ into the two profit functions, we obtain

$$
\begin{aligned}
\pi_{m}^{M}(\cdot, \theta) \equiv & R_{m}^{M}\left(H_{m}, H_{-m}, z\right)-\sum_{h \in H_{m}} x_{m, h}\left(H_{m}, H_{-m}, z\right) \theta-\sum_{h \in H_{m}} \nu_{m, h} \\
\pi_{h}^{H}(\cdot, \theta) \equiv & \sum_{m \in M_{h}} x_{m, h}\left(H_{m}, H_{-m}, z\right) \theta \\
& -c_{h} \sum_{m \in M_{h}} q_{m, h}\left(H_{m}, H_{-m}, z\right)+\sum_{m \in M_{h}} \nu_{m, h} .
\end{aligned}
$$

These equations determine actual (in contrast to measured) realized profits. Our measured variables, $\left(R_{m}^{o}(\cdot), x_{m, h}^{o}(\cdot), q_{m, h}^{o}(\cdot), c_{h}^{o}\right)$ are obtained either directly from data or from a careful study of hospital demand and the formation of HMO premiums described in Ho (2009). We assume that they are correct up to a mean zero measurement error. That is, our measure of profits for HMO $m$ and hospital $h$ given a value for $\theta$ is

$$
\begin{aligned}
& r_{m}^{M}(\cdot, \theta) \equiv R_{m}^{o}(\cdot)-\sum_{h \in H_{m}} x_{m, h}^{o}(\cdot) \theta \\
& r_{h}^{H}(\cdot, \theta) \equiv \sum_{m \in M_{h}} x_{m, h}^{o} \theta-c_{h}^{o} \sum_{m \in M_{h}} q_{m, h}^{o}(\cdot),
\end{aligned}
$$

and our assumptions imply

$$
\begin{aligned}
& r_{m}^{M}(\cdot, \theta) \equiv \mathcal{E}\left[\pi_{m}^{M}(\cdot, \theta) \mid \mathcal{J}_{m}\right]+\mathcal{E}\left[\sum_{h \in H_{m}} \nu_{m, h} \mid \mathcal{J}_{m}\right]+\nu_{1, m, M_{h}, M_{-h}}, \\
& r_{h}^{H}(\cdot, \theta) \equiv \mathcal{E}\left[\pi_{h}^{H}(\cdot, \theta) \mid \mathcal{J}_{h}\right]-\mathcal{E}\left[\sum_{m \in M_{h}} \nu_{m, h} \mid \mathcal{J}_{h}\right]+\nu_{1, h, H_{m}, H_{-m}}
\end{aligned}
$$




\section{Counterfactuals}

Equation (12) provides the profits agents obtain from the observed network. To obtain our moment inequalities, we have to consider the profits, and hence the network, the agents thought would have obtained from counterfactual behavior, and this requires assumptions on the contracting game. Ho's assumptions are both familiar and computationally convenient (we consider a less convenient alternative below). She assumes that sellers simultaneously make take it or leave it offers to buyers, who then simultaneously accept or reject. As in Hart and Tirole (1990) the contract offers are assumed to be proprietary: each HMO knows the offers made to it but not to its competitors, and each hospital knows the offers it makes but not those of its competitors. We observe which HMOs contracted with which hospitals and can compute our measures of returns from any network. To form our moment inequalities, we need to know the network that would be established were either an HMO or a hospital to change its behavior. ${ }^{11}$

The HMOs act last. So our assumptions imply that the HMO could reverse any of its decision without changing the behavior of any other agent. Accordingly our HMO counterfactuals are obtained by reversing the HMO's acceptance-rejection decision with each of the hospitals in the market, leaving all other contracts unchanged, and computing the difference in the HMO's profits between the actual and the counterfactual networks.

To obtain a profit inequality for the hospital, we have to (i) specify an alternative offer the hospital could make and (ii) specify either what the hospital thinks the particular HMO would do were it offered the alternative contract or compute a lower bound to the profits the hospital could make as a result of the actions the HMO might take in response to the alternative contract. We assume that the hospital could always offer a null contract (a contract which is never accepted). What the hospital thinks the HMO would do if offered this contract depends on how the hospital thinks receiving the alternative contract would affect the HMO's beliefs about the contracts offered to other hospitals, and given those beliefs, whether the hospital thinks the HMO would change its replies to the contracts offered by other hospitals. We assume "passive beliefs," that is, the hospital believes that the HMO will not change its beliefs about the offers the hospital makes to other HMOs were it to receive the counterfactual offer, and present results which assume that the hospital thinks the HMO would not change its behavior with other hospitals were it to receive the null contract. However, we have also done the analysis assuming the hospital thinks the HMO might add a different hospital with little difference in empirical findings.

\footnotetext{
${ }^{11}$ Since we assume that the premium setting game is a full information game, our assumptions are what McAfee and Schwartz (1992) refer to as "ex poste observability"; the HMOs do not know each other's offer in the first period, but the costs in each accepted contract are revealed before the second stage of the game. This assumption could be relaxed at a cost of increasing the computational burden of estimation.
} 


\section{Inequalities Used}

We began with Ho's assumption that $\mathcal{E}\left[\nu_{m, h} \mid \mathcal{J}_{i}\right]=0$ for $i=(m, h)$. Then the only disturbances in equations (13) are $\nu_{1}$ disturbances, so we can form our inequalities by interacting positive functions of variables that were known to the decision maker when the decision was made with the difference between our models' estimates of the profits actually earned and those that would have been generated by our counterfactuals. Recall that these are the HMO's profits from reversing its decision with each hospital and the hospital's profits from offering a null contract to an HMO which had accepted its offer.

Next we considered alternative ways to allow $\nu_{m, h}$ to have a $\nu_{2}$ component, that is, we allowed $\mathcal{E}\left[\nu_{m, h} \mid \mathcal{J}_{i}\right] \equiv \nu_{2, m, h} \neq 0$ and the same value for $i=m, h$. We first tried $\nu_{2, m, h}=\nu_{2, m} \forall(m, h)$; that is, that the $\nu_{2}$ are HMO-specific fixed effects. As shown in the Appendix, we can then use PC4a to generate a quite detailed set of inequalities. There is no a priori reason to assume a fixed effects structure here: when we did, it accentuated the problems with the $\nu_{1}$-only model, ${ }^{12}$ so we used the generalized version of PC4b in footnote 10 to develop an estimator for the buyer-seller network problem that allows for a $\nu_{2, m, h}$ of a general form.

Recall that the $\nu_{m, h}$ are a component of transfers, so the same $\nu_{m, h}$ value that goes into a hospital's revenues is a component of an HMO's costs. Let $\chi_{m, h}$ be the indicator function for whether a contract is established between $m$ and $h$ with $\chi_{m, h}=1$ if it is established and 0 if not. These are the only two outcomes possible. So to satisfy PC4b, we need an inequality which is additively separable in $\nu_{m, h}$ regardless of whether $\chi_{m, h}=0$ or 1 .

Let $\Delta \pi_{h}^{H}\left(M_{h}, M_{h} / m, M_{-h}, z\right)$ be the difference between the hospital's profit when the network of the hospital includes HMO $m$ and when it does not. If $\chi_{m, h}=1$, this contains $\nu_{m, h}$. Let $\Delta \pi_{m}^{M}\left(H_{m}, H_{m} \cup h, H_{-m}, z\right)$ be the difference between the HMO's profit were it to reject hospital $h$ 's contract and were it to accept it. If $\chi_{m, h}=0$, this includes the savings in $\nu_{m, h}$ from rejecting the contract. Note that if $\chi_{m, h}=1, \mathcal{E}\left[\Delta \pi_{h}^{H}\left(M_{h}, M_{h} / m, M_{-h}, z\right) \mid \mathcal{J}_{h}\right] \geq 0$, while if $\chi_{m, h}=0, \mathcal{E}\left[\Delta \pi_{m}^{M}\left(H_{m}, H_{m} \cup h, H_{-m}, z\right) \mid \mathcal{J}_{m}\right] \geq 0$. Using analogous notation for $\Delta r(\cdot)$, equation (13) implies

$$
\begin{aligned}
& \chi_{m, h} \Delta r_{h}^{H}\left(M_{h}, M_{h} / m, M_{-h}, z ; \theta\right) \\
& \quad+\left(1-\chi_{m, h}\right) \Delta r_{m}^{M}\left(H_{m}, H_{m} \cup h, H_{-m}, z ; \theta\right)
\end{aligned}
$$

\footnotetext{
${ }^{12}$ In the $\nu_{1}$-only model, about $12 \%$ of the inequalities were negative, but under $2 \%$ were individually significant at the $5 \%$ level. In the model with fixed effects, about a third of the inequalities were negative and $10 \%$ were significant at the $5 \%$ level. A more complete analysis of effects models in buyer-seller networks would allow for both buyer and seller effects. This is a straightforward, although somewhat tedious, extension of the results in the Appendix. We examine the HMO effects case in detail because all the contract correlates we use in our analysis are hospitalspecific and we wanted to make sure that the absence of HMO characteristics did not bias the analysis of the impacts of these hospital-specific variables.
} 


$$
\begin{aligned}
= & \chi_{m, h}\left(\mathcal{E}\left[\Delta \pi_{h}^{H}\left(M_{h}, M_{h} / m, \cdot\right) \mid \mathcal{J}_{h}\right]+\nu_{2, m, h}+\Delta \nu_{1, m, .}\right) \\
& +\left(1-\chi_{m, h}\right)\left(\mathcal{E}\left[\Delta \pi_{m}^{M}\left(H_{m}, H_{m} \cup h, \cdot\right) \mid \mathcal{J}_{m}\right]+\nu_{2, m, h}+\Delta \nu_{1, h, .}\right) \\
= & \chi_{m, h}\left(\mathcal{E}\left[\Delta \pi_{h}^{H}\left(M_{h}, M_{h} / m, \cdot\right) \mid \mathcal{J}_{h}\right]+\Delta \nu_{1, m,}\right) \\
& +\left(1-\chi_{m, h}\right)\left(\mathcal{E}\left[\Delta \pi_{m}^{M}\left(H_{m}, H_{m} \cup h, \cdot\right) \mid \mathcal{J}_{m}\right]+\Delta \nu_{1, h,}\right)+\nu_{2, m, h},
\end{aligned}
$$

which is additive in $\nu_{2, m, h}$ regardless of whether $\chi_{m, h}$ is 1 or 0 . So there is no selection and provided $x \in \mathcal{J}_{m} \cap \mathcal{J}_{h}, E\left[\nu_{2, m, h} \mid x\right]=0$, and $h(\cdot) \geq 0$, PC3 insures that at $\theta=\theta_{0}$,

$$
\begin{aligned}
& E\left(\chi_{m, h} \Delta r_{h}^{H}\left(M_{h}, M_{h} / m, M_{-h}, z ; \theta\right)\right. \\
& \left.\quad+\left(1-\chi_{m, h}\right) \Delta r_{m}^{M}\left(H_{m}, H_{m} \cup h, H_{-m}, z ; \theta\right)\right) h(x) \geq 0 .
\end{aligned}
$$

The model also delivers an inequality that does not depend on the $\nu_{m, h}$ (as in PC4a). The sum of the increments in profits to the HMO and the hospital when a contract is established does not contain the transfers between them (and hence $\nu_{m, h}$ ), does contain information on $\theta$ (since if the contract is not established there is a change in transfers to other agents), and must have positive expectation (at least if contract offers are proprietary). So if $H_{m} / h$ is the observed network of hospital $m$ minus hospital $h$, our conditions on $x$ also insure

$$
E\left[\chi_{m, h}\left(\Delta r_{m}^{M}\left(H_{m}, H_{m} / h, \cdot ; \theta_{0}\right)+r_{h}^{H}\left(M_{h}, M_{h} / m, \cdot ; \theta_{0}\right)\right) h(x)\right] \geq 0 .
$$

\section{Estimates}

Neither the $\nu_{1}$-only nor the model which allowed for $\nu_{2}$ could be rejected by our formal tests. This is not surprising given the sample size. ${ }^{13}$ However, the results did seem to favor the model that allows for $\nu_{2}$, as only 6 of the inequalities were negative at its estimated parameter value (the $\nu_{1}$-only model

\footnotetext{
${ }^{13}$ There were 40 markets containing about 450 plans and 630 hospitals. The market characteristics used as instruments were indicators for the quartile of the market's population size, high (greater than mean) share of population aged 55-64, and hospitals integrated into systems. The plan characteristics were indicators for whether the plan was local, its quartile of the breast screening distribution, the quality of its mental health services, and an interaction between the last two variables. The hospital cost measure was not used as an instrument because we were worried about measurement error in that variable. The results reported here weighted the market averages of the moment inequalities by the square root of the number of plans in the market, as this produced slightly smaller confidence intervals (interestingly weighting by the variance of the moment inequalities did not improve those intervals). Confidence intervals for each dimension are computed using the techniques in Pakes et al. (2006); a Monte Carlo study of their properties is available from the author). Finally, Ho (2009) reported a series of robustness checks on the $\nu_{1}$ only estimates of a model which is similar to the model presented here. Although specifications which add right-hand side variables sometimes increase the confidence intervals quite a bit, the qualitative results in our column 1 in Table I are never reversed.
} 
TABLE I

DETERMINANTS OF HOSPITAL-HMO CONTRACTS ${ }^{\mathrm{a}}$

\begin{tabular}{|c|c|c|c|c|c|c|c|c|}
\hline \multirow{5}{*}{$\begin{array}{r}\text { Data: } \\
\text { Estimator: } \\
\text { Column: }\end{array}$} & \multicolumn{4}{|c|}{ Real Datab } & \multicolumn{4}{|c|}{ Simulated Data ${ }^{\mathrm{c}}$} \\
\hline & \multicolumn{4}{|c|}{ Inequality Estimators } & \multirow{2}{*}{\multicolumn{4}{|c|}{$\begin{array}{l}\text { OLS Regression } \\
\text { Actual Markups }\end{array}$}} \\
\hline & \multicolumn{2}{|c|}{$\nu_{1}$-Only } & \multicolumn{2}{|c|}{$\nu_{1}$ and $\nu_{2}$} & & & & \\
\hline & (1) & (2) & (3) & (4) & (5) & (6) & (7) & (8) \\
\hline & $\theta$ & $95 \% \mathrm{CI}$ & $\theta$ & $95 \% \mathrm{CI}$ & $\theta$ & s.e. & $\theta$ & s.e. \\
\hline Variable & & $\mathrm{UB} / \mathrm{LB}$ & & $\mathrm{UB} / \mathrm{LB}$ & & & & \\
\hline \multicolumn{9}{|c|}{ Per patient markup (units $=\$$ thousand $/$ patient) } \\
\hline Const. & 9.5 & $15.4 / 4.8$ & 8.2 & $15.2 / 3.3$ & 8.9 & 0.09 & 3.7 & 0.24 \\
\hline CapCon & 3.5 & $8.6 / 1.4$ & 13.5 & $16.1 / 2.3$ & 1.2 & 0.10 & 0.48 & 0.11 \\
\hline Cost/Adm. & -0.95 & $-1.5 /-0.57$ & -0.58 & $-0.2 /-1.1$ & -0.39 & 0.01 & - & - \\
\hline Av. Cost & - & - & - & - & - & - & -0.23 & 0.01 \\
\hline Cost $-\mathrm{AC}$ & - & - & - & _- & - & - & -0.56 & 0.01 \\
\hline Pop/bed & - & - & - & - & - & - & 0.11 & 0.01 \\
\hline \# Patients & - & - & - & - & - & - & -0.09 & 0.01 \\
\hline HMOmarg & - & - & - & - & - & - & 1.4 & 0.10 \\
\hline$R^{2}$ & - & - & - & - & \multicolumn{2}{|c|}{0.71} & \multicolumn{2}{|c|}{0.80} \\
\hline
\end{tabular}

${ }^{a}$ Notation: CI, confidence interval; s.e., standard error; UB, upper bound; LB, lower bound.

${ }^{b}$ Real Data: There are 40 markets. CapCon measures whether the hospital would be capacity constrained if all hospitals contracted with all HMOs. Cost/Adm denotes hospital cost per admission. Costs and admissions are not elements of instrumental variables (IV).

${ }^{\mathrm{c}}$ Simulated Data: These are least squares regression coefficients from projecting computed markups onto the included variables. See below for the calculation of equilibrium markups. There are 1,385 markets with two HMOs and two hospitals in each. This generates approximately the same number of buyer-seller pairings as in the data set used in the empirical analysis. Additional variables are defined as follows: Cost - AC is the cost per admission of the hospital minus the average of that over the hospitals in the market, Pop/bed is population over total number of hospital beds in the market, \# Patients is number of patients the HMO sends to the hospital, and HMOmarg is the HMO's average premium minus its average cost.

had 11), and none of them as individually significant at the $5 \%$ level (in contrast to 1 for the $\nu_{1}$-only model).

The first four columns of Table I present the empirical results. We subtracted our estimate of hospital costs from the revenues in all specifications, so the coefficients appearing in the table are the coefficients of the markup implicit in the per patient payment. Despite the fact that none of the test statistics we computed was significant at the $5 \%$ level, there was no value of $\theta$ which satisfied all the inequality constraints in any specification, a finding that is not unusual when there are many inequalities (all our specifications had 88 or more of them). The algorithm then generates a point estimate equal to the $\theta$ value that minimizes a squared metric in the negative part of the sample moments.

Sample size limited the right-hand side variables we could use in the investigation. Still the estimates we do get, though reduced form, are eye-opening. They imply an equilibrium configuration in which the majority of cost savings from low cost hospitals are captured by the HMOs, and markups increase sharply when a hospital is capacity constrained (CapCon measures whether 
the hospital would be capacity constrained if all hospitals contracted with all HMOs). Although these are not structural estimates, they do lead us to worry about the possibility of significantly lower incentives for hospitals to invest in either cost savings or in capacity expansion than would occur in a price-taking equilibrium. The difference between the $\nu_{1}$-only estimates and those that allow for $\nu_{2}$ is that the former imply that almost all the cost savings from low cost hospitals go to the HMOs, while the latter imply that just over 50\% do and a larger fraction of profits go to capacity constrained hospitals. Low cost hospitals tend to be more capacity constrained, so the two variables are negatively correlated.

\subsection{Numerical Analysis}

Might we expect contracts with these characteristics to emanate from a contracting equilibrium and should we interpret those coefficients to mean that an increase in the right-hand side variable would, ceteris paribus, generate the markup response we estimate? To shed some light on these issues, we computed equilibria to a structural contracting model in markets with characteristics similar to those in Ho's data, but with population scaled down to a size where we would expect to have two hospitals and two HMOs in each market (this made it possible to compute equilibria for many markets in a reasonable amount of time). ${ }^{14}$

We compute a full information Nash equilibrium to a game in which hospitals make take it or leave it offers to HMOs. The algorithm assumes that both hospitals choose among a finite set of couples of markups, one for each HMO, and that these markups are offered simultaneously to the HMOs. The offers are public information, as are the HMO premiums that would result from any set of contracts (these are obtained as the Nash equilibrium to a premium setting game among the HMOs). The HMOs then simultaneously accept or reject the offers. At equilibrium, each hospital is making the best offers it can given the offers of the other hospital and the responses of the HMOs, and each HMO is doing the best it can do given the actions of its competitor and the offers made by the hospitals. ${ }^{15}$

\footnotetext{
${ }^{14} \mathrm{We}$ used a discrete choice model of demand and market characteristics determined by random draws from demand and cost characteristic distributions that mimicked those in Ho's data. The closest exercise I know of is in a paper by Gal-Or (1997). By judicious choice of primitives, she is able to provide analytic results from a full information Nash bargaining game between two HMOs and two hospitals. She focuses on when her assumptions would generate exclusive dealing and its effects on consumers.

${ }^{15}$ A more detailed description of the algorithm can be found in Lee and Pakes (2010). An iterative process with an initial condition in which both hospitals contract with both HMOs chooses among the equilibria when there are multiple equilibria. The choice set included 50 possible markups for each of the two hospitals. The algorithm starts with the lowest ones. It then determines whether HMO1 wants to reject one (or both) of the contracts conditional on HMO2
} 
Note that these assumptions differ from those used in the empirical analysis. In this full information game, the necessary conditions for an equilibrium guarantee an outcome which is renegotiation-proof, while the necessary conditions for the asymmetric information game we took to data do not. The related questions of (i) when the different equilibrium notions are appropriate and (ii) whether the estimation results are sensitive to this choice are questions that research on buyer-seller networks will have to sort out. Although the contents of contracts are often proprietary, typically who contracts with whom is not. So if we were trying to model a set of relationships which have been stable over some time, we might only consider equilibria in which no two agents would find it profitable to recontract given the information on who is contracting with whom. Of course the market we are studying may be constantly changing and negotiations might be costly. Then we might not expect the data to abide by a renegotiation-proof criteria, at least not one with costless renegotiation. Since all we need for estimation is a way to obtain a lower bound to the expected profits from a counterfactual choice, we could, at least in principle, obtain our inequalities from the difference between the actual profits and the minimum of the profits from a group of counterfactuals chosen to reflect different possible game forms (although the larger the group, the less tight our bounds and the larger the computational burden).

\section{Numerical Results}

Columns 5-8 of Table I present ordinary least squares (OLS) estimates from regressing the computed markups onto variables of interest. The first two columns show that the three variables that the empirical study focused on have the appropriate signs, are significant, and account for a large fraction, about $70 \%$, of the variation in markups (or about $85 \%$ of the variance in transfers). Columns 7 and 8 add variables. The original three variables maintain their signs and remain significant but have noticeably different magnitudes; that is,

being contracted to both hospitals. This requires solving for equilibrium premiums and profits for HMO1 given each possible choice it can make and the fact that HMO2 is contracted to both hospitals. HMO2 then computes its optimal responses to HMO1's decisions in the same way. This process is repeated until we find a Nash equilibrium for the HMOs' responses. No matter the offers, we always found an equilibrium to this subgame. We then optimize over the first hospital's (say H1) offers, holding H2's offers fixed. For each offer, we repeat the process above until we find a Nash equilibrium for the HMOs' responses. This gives us H1's optimal offers given the initial offers by H2. Next H1's offers are held fixed and H2's offers are optimized against that. We repeat this process until we find a Nash equilibrium in offers. For 3\% of the random draws of characteristics, we could not find an equilibria and those markets were dropped from the analysis. Note that when a hospital contracts with an HMO in equilibrium, it does not necessarily contract at the lowest offer that is consistent with the HMO accepting. Different offers change the HMO costs per patient. This changes the outcome of the premium setting game that the HMOs engage in and feeds back into hospital profits. 
although the empirical results do pick up important correlates of the equilibrium payments, the reduced form parameter estimates should not be thought of as causal responses.

The coefficients of the additional variables in column 7 are instructive. They imply that when the average hospital cost in the market goes up by $1 \%$, the markups of the hospitals in the market go down by $23 \%$, but if the difference between a hospital's cost and the average hospital cost goes up by $1 \%$, the hospital's markup goes down by $56 \%$. So a hospital's markup over costs depends on the costs of the other hospitals it is competing with. Hospitals earn higher markups in "tighter" markets (markets with lower ratios of population to the number of hospital beds) and once we account for this, the effect of capacity constraints is greatly reduced (though not eliminated). HMOs seem to get a small quantity discount (the markups they pay are lower when they send more patients to the hospital), and hospitals earn higher markups when the HMOs they are dealing with charge their members higher markups.

Finally note that $20 \%$ of the variance in markups, or $8 \%$ of the variance in transfers, is not accounted for by our observables. Given the full information assumptions, this is $\nu_{2}$ variance. Even in a world where our equilibrium and functional form assumptions are correct, measurement error in hospital costs would cause $\nu_{1}$ error. So in this (and we suspect in most) empirical example, both types of errors are likely to be present.

\section{SPECIFICATION ERRORS AND ALTERNATIVE ESTIMATORS}

The generalized discrete choice model ignores $\nu_{1}$ errors and requires an a priori specification of the $\nu_{2}$ distribution, both assumptions which, if incorrect, can generate an inconsistency in its estimators. The profit inequality model which pays inadequate attention to possible sources of $\nu_{2}$ error will generate selection biases. This section asks what the impacts of these specification errors are likely to be in the context of our buyer-seller network example. It presents Monte Carlo results from using each of the two model's estimators both (i) when that models' assumptions are the assumptions generating the data and (ii) when they are not. Where possible, we will also present results from Ho's data.

\section{Details of the Monte Carlo Analysis}

The Monte Carlo results are based on a population of 100,000 markets whose equilibria were computed using the algorithm described in the last section. We estimate one parameter; the average per patient markup. To obtain the true value of that parameter, we took the transfers implicit in the equilibrium offers and projected them onto the number of patients and the variables 
we used as instruments. ${ }^{16}$ The function obtained from this projection is treated as the parametric transfer function. The coefficients of the instruments are treated as known and the coefficient of the patient variable is the coefficient to be estimated. The residual from this projection is the $\nu_{2}$ error. This insures that $\nu_{2}$ has zero covariance with our instruments before we condition on the outcome. When all we require is a $\nu_{1}$ error, we treat these $\nu_{2}$ as known and add pseudorandom draws on a normal measurement error to hospital costs and/or population size.

The Monte Carlo results are based on 400 data sets, each obtained as independent draws from our "population" of markets. The sample size was set so that the number of contracts in each sample matched the number of contracts in Ho's (2009) data set. Since each of the computed equilibria had only two hospitals and two HMOs, this gave us a larger number of markets (1,385 markets per sample), but many fewer contracts per market, than in Ho (2009). The $\nu_{1}$ and $\nu_{2}$ draws are taken independently across samples. ${ }^{17}$

The inequalities used to estimate the profit inequality model are the same as those used in the empirical work: each HMO reverses its equilibrium decision with each hospital and each hospital replaces its equilibrium contract offer to each HMO with a null contract. However, since the Monte Carlo data are generated from a full information Nash equilibrium, when the hospital offers a null contract to an HMO, that hospital considers the profits that would accrue to it were both HMOs to reoptimize. ${ }^{18}$ For the generalized discrete choice approach, we used the inequalities generated by the necessary conditions for equilibrium. ${ }^{19}$

\section{Results}

Table II, which presents the results, is split into panels. Panel A provides estimates obtained using the $\nu_{1}$-only inequalities, panel $\mathrm{B}$, using the $\nu_{2}$-only

\footnotetext{
${ }^{16}$ For accepted offers, these were the actual transfers; for the offers that were rejected, these are the transfers that would have resulted if the last offer had been accepted.

${ }^{17}$ Actually we did the analysis in two ways. In the second, we drew a Monte Carlo data set, took 200 draws on vectors of $\nu_{1}$ errors for that data set, tabulated the results for each data set, and then averaged over data sets. This provides confidence intervals that condition on the observables, while the results reported in the text do not. The results from the two procedures were virtually identical.

${ }^{18} \mathrm{To}$ obtain the $\pi(\cdot)$ resulting from the null contract offer, let $o_{m, h}$ be the contract offered by hospital $h$ to HMO $m$ in equilibrium, and let $\phi$ be the null contract. If $h=1$ contracted with $m=1$, its profits from offering $\phi$ are obtained from the HMO equilibrium responses to the tuple $\left(\phi, o_{1,2}, o_{2,1}, o_{2,2}\right)$.

${ }^{19}$ Note that the estimating equations used do not exhaust the information in the data in either approach. At the cost of increasing the computational burden, we (i) could have used the inequalities obtained from simultaneously switching each HMO's behavior with respect to both hospitals in the profit inequality approach (and if more details on the contracts were available yet other inequalities would become available), and (ii) for the generalized discrete choice approach, we could have computed the probability that the observed equilibrium was unique.
} 
TABLE II

INEQUALITY ESTIMATORS: SIMULATED AND ACTUAL DATA ${ }^{\mathrm{a}}$

\begin{tabular}{lllllll}
\hline \hline \multirow{2}{*}{ Disturbances } & Not in IV & & \multicolumn{2}{c}{ Average } & & \multicolumn{2}{c}{$95 \%$ of $\theta$ in } \\
& & & LB & UB & & UB \\
\hline
\end{tabular}

A. Using $\nu_{1}$ inequalities

Simulated data: $\theta_{0}=16.76,\left[\underline{\theta}_{0}, \bar{\theta}_{0}\right]=[13.47,18.59]$

Only $\nu_{1}$ disturbances

\begin{tabular}{lccccc} 
1. $25 \%$ Cost & Cost & 12.39 & 18.72 & 12.12 & 19.05 \\
$\begin{array}{l}\text { 2. } 25 \% \text { Cost, 5\% pop } \\
\nu_{1} \text { and } \nu_{2} \text { disturbances }\end{array}$ & Cost, $N_{j, k}$, pop & 11.43 & 37.34 & 11.30 & 45.88 \\
$\begin{array}{l}\text { 3. } \nu_{2} \text {, Costs } \\
\text { 4. } \nu_{2} \text {, Costs, pop }\end{array}$ & Cost & 12.25 & 18.42 & 12.01 & 18.86 \\
$\begin{array}{l}\text { Ho's (2009) data with } \nu_{1} \text { inequalities } \\
\text { 5. Actual disturbances }\end{array}$ & Cost, $N_{j, k}$, pop & 11.69 & 35.91 & 11.55 & 43.97 \\
& Cost & 8.2 & 8.2 & 2.3 & 16.4 \\
\hline
\end{tabular}

B. Using $\nu_{2}$ inequalities

Simulated data, only constant as IV: $\theta_{0}=16.76,\left[\underline{\theta}_{0}, \bar{\theta}_{0}\right]=[16.7,16.95]$

$\begin{array}{lllll}\text { 6. } \nu_{2} \text { (bootstrap dist.) } & 17.75 & 17.75 & 17.25 & 18.1 \\ \text { 7. } \nu_{2} \text { (normal dist.) } & 17.92 & 17.92 & 17.2 & 18.45\end{array}$

Simulated data, all IV: $\theta_{0}=16.76,\left[\underline{\theta}_{0}, \bar{\theta}_{0}\right]=[16.7,16.85]$

8. $\nu_{2}$ (bootstrap dist.)

$\begin{array}{llll}17.84 & 17.84 & 17.40 & 18.25\end{array}$

$\begin{array}{lllll}\text { 9. } \nu_{2} \text { (normal dist.) } & 18.02 & 18.02 & 17.65 & 18.5\end{array}$

Simulated data, $\nu_{1}$ (in costs) and $\nu_{2}$ disturbances

$\begin{array}{lllll}\text { 10. } \nu_{2} \sim \mathcal{N}, \text { IV }=\text { only constant } & 18.02 & 18.02 & 17.35 & 18.5\end{array}$

$\begin{array}{llllll}\text { 11. } \nu_{2} \sim \mathcal{N} \text {, all IV } & \text { Costs } & 18.11 & 18.11 & 17.64 & 18.65\end{array}$

Ho's (2009) data with $\nu_{2}$ inequalities

12. Assume $\nu_{2}$ normal Could not compute

Simulated data

C. Using robust inequalities
13. $\nu_{2}$, Costs
14. $\nu_{2}$, Costs, pop
$\begin{array}{cr}\text { Cost } & 11.86 \\ \text { Cost, } N_{j, k}, \text { pop } & 11.69\end{array}$
n.b. $\quad 11.72$
n.b.

Ho's (2009) data

15. Actual disturbances

Cost

11.7

11.7

3.6

17.9

\footnotetext{
${ }^{\mathrm{a}}$ Instruments for panels A and C (unless omitted) are constant, $N_{j, k}$, hospital cost and capacity measures, market cost capacity and population measures, HMO characteristics, and interactions among them. Instruments for $\nu_{2}$ inequalities are market averages of the above variables. The model for line 15 also allowed for a cost coefficient; without it the average markup was negative.
}

inequalities (the inequalities from the generalized discrete choice model), and panel $\mathrm{C}$ using the inequalities that allow for both $\nu_{1}$ and $\nu_{2}$ disturbances. The "true" value of $\theta_{0}$ from the simulated data was 16.76 . The "identified set," that is, the $\theta$ interval that satisfies the population moment conditions, differs across panels.

Since the instruments are orthogonal to the disturbance by construction, the identified set for panel $\mathrm{A}$ is the $\theta$ interval which generates positive popula- 
tion profit inequalities when we set all disturbances to zero: [13.47, 18.59]. The true identified set for the generalized discrete choice model depends on the true joint distribution of the $\nu_{2}$ 's conditional on the market's instruments. This is not known and is too complex to estimate nonparametrically-a problem which is likely to recur in empirical work. To get a sense of the identified set generated by this approach, we set all the disturbances to zero and, for each possible network structure, found the set of $\theta$ which lead to positive values for (i) the averages of the differences between the indicator functions for satisfying the Nash conditions and the observed equilibrium outcome, and (ii) did the same after interacting the difference in indicator functions for each network structure with the variables used as instruments. The interval for (i) was [16.7, 16.95], while for (ii) it was [16.7, 16.85]—both rather amazingly short.

The first two rows of panel A provide results from the $\nu_{1}$-only profit inequality model when there are only $\nu_{1}$ errors (so its estimators are consistent). Row 1 adds measurement error in costs equal to $25 \%$ of the true cost variance. The average of the estimated lower bounds is $8 \%$ lower than the true lower bound $\left(\underline{\theta}_{0}\right)$, while that of the upper bound is $2.5 \%$ higher than $\bar{\theta}_{0}$. Moreover, the bounds are precisely estimated, less than $2.5 \%$ of the lower (upper) bounds were more than $10 \%$ different from their true values. When we add an expectational error to the population, and hence to the patient flows from the HMOs to the hospitals, the estimated interval gets substantially larger. This is unfair to the model since, although there may be uncertainty in the relevant population size and patient flows variables when contracts are signed, we should be able to construct good instruments for them from current population size and flows, and we did not do that here. We keep this case because it allows us to examine the impact of specification errors in one setting where the bounds define a short interval and one where they do not.

Rows 3 and 4 use a simulated data set that contains both $\nu_{1}$ and $\nu_{2}$ errors but the inequalities from the $\nu_{1}$-only model. The ratio of the variance in $\nu_{2}$ to the variance in the dependent variable is $12.7 \%$. Now the estimated bounds are inconsistent; the lower bound will, in the limit, be too large, while the upper bound will be too low. This makes the bounds move toward $\theta_{0}$, but they may overshoot, leaving us with an estimator which does not cover the true $\theta_{0}$. Adding $\nu_{2}$ also adds variance to the estimators, so in any finite sample, the estimated bounds may be smaller or larger with $\nu_{2}$ errors than without them. In the case with only measurement error in costs, the case in which the interval was tightly estimated, adding specification error in the form of the $\nu_{2}$ has little effect on any of the estimates. When there is also measurement error in population and the estimated intervals are larger, the effect of the specification error is to lessen the loosely estimated upper bound, but only by $5 \%$. At least in this example, estimates from the $\nu_{1}$-only inequalities do not change much when we allowed for $\nu_{2}$ error. Apparently when we add $\nu_{2}$ variance, its biasing effects on the estimates are largely offset by the effect of increased data variance on those estimates. 
The last row of panel A provides the estimates when we use Ho's (2009) data with this specification. This generates a point estimate about a third lower than the lower bound estimate from the simulated data, and a confidence interval of length between that of the model with errors in the population and that without those errors.

Panel B provides the results when we use the $\nu_{2}$-only inequalities. To use the $\nu_{2}$-only algorithm, we need an assumption on the joint distribution of the $\nu_{2}$. We tried two assumptions: (i) random draws from the empirical distribution of the actual $\nu_{2}$ and (ii) a normal distribution. The first option would not be available to empirical researchers, but might be closer to the true population distribution (it would be if the $\nu_{2}$ were truly independent, not just mean independent, of the instruments and had no within market correlation).

Regardless of whether we use just the constant term (rows 6 and 7) or all of our instruments (rows 8 and 9) and regardless of the choice of $\nu_{2}$-distribution, the $\nu_{2}$-only model generates point estimates whose values are larger than the true $\theta_{0}$. The distribution of estimates had little variance, so the interval formed from $95 \%$ of the point estimates does not cover the true $\theta_{0}$ either. Apparently the lack of information on the $\nu_{2}$-distribution leads to an inconsistency. Although this is disturbing, the asymptotic bias is not large; the lower bound of the (normal) confidence interval is about $2.6 \%$ larger and the point estimate is $6.9 \%$ larger than $\theta_{0}$.

Just as we added $\nu_{2}$ variance to the algorithm which uses the $\nu_{1}$-only inequalities, rows 10 and 11 add $\nu_{1}$ variance to the algorithm which uses the $\nu_{2}$-only inequalities. The estimates presented in these rows use the normal distribution of the $\nu_{2}$ (an empirical researcher would not have access to the bootstrap distribution). Adding $\nu_{1}$ errors does tend to increase the parameter estimates further, but by a surprisingly small amount. We could not use the $\nu_{2}$-only algorithms on Ho's actual data. To do so we would have had to compute about 100,000 premium setting equilibria and their implied profits for each $\nu_{2}$ draw and each $\theta$ evaluated in estimation-a task that will be beyond our computational abilities for some time.

Panel $\mathrm{C}$ provides the estimates obtained when we used the inequalities that allow for both $\nu_{1}$ and $\nu_{2}$ disturbances-the "robust" inequalities in equations (14) and (15). The fact that there are only two agents on each side of the simulated markets implies that the robust inequalities do not deliver an upper bound. The lower bound is lower than the bound obtained when we used the $\nu_{1}$-only inequalities on this data, but not by much. When we move to Ho's (2009) data and use the robust inequalities, we get an estimate which is larger than the estimate which allows for only $\nu_{1}$ errors but a confidence interval of similar length, and both confidence intervals cover both estimates. Interestingly, once we allow for $\nu_{2}$ errors, the estimates from Ho's data are closer to, and the confidence interval covers, the value of the parameter obtained from the numerical analysis. 
The results from the Monte Carlo analysis are quite encouraging. It seems that the most salient problem is the requirement of an assumption on the distribution of $\nu_{2}$ in the generalized discrete choice model. In multistage games, that estimator also carries a large computational burden. However, the worry that a moderate amount of $\nu_{1}$ variance in the generalized discrete choice model or a moderate amount of $\nu_{2}$ variance in the profit inequality model would severely bias the estimates is, at least in this example, not warranted. The addition of the unaccounted for error adds variance, as well as bias, to the estimates. This variance tends to move the bounds in the opposite direction as does the bias, and in our example, the net effect was small. The estimator which uses the robust inequalities is least subject to bias but does generate larger identified sets.

\section{SUMMARY}

This paper formulates two sets of assumptions that enable one to bring behavioral models - both their structural and their reduced forms - to data and applies them to two empirical problems. Our first example illustrates that the assumptions underlying traditional discrete choice estimators are not always the most sensible choice for discrete choice problems. This motivates an enumeration of assumptions that justify alternative estimators in both multiple and single agent settings. An empirical example illustrates how the multiple agent estimator can be used to analyze a problem which is central to the determinants of prices and investment incentives in vertical markets; the correlates of the profit split between buyers and sellers in those markets. Although the results were reduced form and had to make do with both limited data and the auxiliary assumptions required to obtain counterfactual profits, they were broadly consistent with results obtained from a numerical analysis of equilibrium contracts in markets which were similar to those used in the empirical analysis. A Monte Carlo analysis indicated that the estimators from both models were surprisingly robust to all likely sources of problems but one: the need to assume a distribution for the generalized discrete choice model. It seems that moment inequalities open up possibilities for empirically analyzing market interactions in relatively unexplored, yet important, settings.

\section{APPENDIX: INEQUALITIES FOR BUYER-SELLER NETWORK WITH FIXED EFFECTS}

We use the notation introduced for the hospital-HMO problem in Section 3.1 and consider the case in which the $\left\{\nu_{2, m, h}\right\}$ are HMO fixed effects; that is, that $\forall(h, m), \nu_{2, m, h}=\nu_{2, m}$. These restrictions generate two sets of inequalities.

The first is a difference in difference inequality. If an HMO accepts at least one hospital's contract and rejects the contract of another, then the sum of 
the increment in profits from accepting the contract accepted and rejecting the contract rejected (i) differences out the HMO effect and (ii) has a positive expectation. More formally, for every $\tilde{h} \notin H_{m}$ and $h \in H_{m}$, we have

$$
\begin{aligned}
& \Delta \pi_{m}^{M}\left(H_{m}, H_{m} \cup \tilde{h}, \cdot\right)+\Delta \pi_{m}^{M}\left(H_{m}, H_{m} \backslash h, \cdot\right) \\
& \quad=\Delta r_{m}^{M}\left(H_{m}, H_{m} \cup \tilde{h}, \cdot\right)+\Delta r_{m}^{M}\left(H_{m}, H_{m} \backslash h, \cdot\right),
\end{aligned}
$$

which implies that provided $x \in \mathcal{J}_{m} \cap \mathcal{J}_{h}$ and $h(\cdot)$ is a positive valued function,

$$
E\left[\Delta r_{m}^{M}\left(H_{m}, H_{m} \cup \tilde{h}, \cdot ; \theta_{0}\right)+\Delta r_{m}^{M}\left(H_{m}, H_{m} \backslash h, \cdot ; \theta_{0}\right)\right] h(x) \geq 0 .
$$

For the second inequality, note that if $\nu_{2, m, h}=\nu_{2, m}$ we can use the logic leading to equation (14) in the text to show that for any positive valued function $h(\cdot)$,

$$
\begin{aligned}
0 \leq & E\left[\frac { 1 } { \# H } \sum _ { h } \left(\chi_{m, h} \Delta \pi_{h}^{H}\left(M_{h}, M_{h} / h, \cdot\right)\right.\right. \\
& \left.\left.+\left(1-\chi_{m, h}\right) \Delta \pi_{m}^{M}\left(H_{m}, H_{m} \cup h, \cdot\right)\right)\right] h(x) \\
= & E\left[\frac { 1 } { \# H } \sum _ { h } \left(\chi_{m, h} \Delta r_{h}^{H}\left(M_{h}, M_{h} / h, \cdot ; \theta\right)\right.\right. \\
& \left.\left.+\left(1-\chi_{m, h}\right) \Delta r_{m}^{M}\left(H_{m}, H_{m} \cup h, \cdot ; \theta\right)\right)+\nu_{2, m}\right] h(x) \\
\equiv & E\left[\bar{S}^{r}(m, \cdot ; \theta)+\nu_{2, m}\right] h(x) .
\end{aligned}
$$

This implies that $E \bar{S}^{r}\left(m, \cdot ; \theta_{0}\right) h(x) \geq-E \nu_{2, m} h(x)$ and, consequently, that for any $x \in \mathcal{J}_{m} \cap \mathcal{J}_{h}$,

$$
E\left[\Delta r_{m}^{M}\left(H_{m}, H_{m} \backslash h, \cdot ; \theta_{0}\right)+\bar{S}^{r}\left(m, \cdot ; \theta_{0}\right)\right] h(x) \geq 0 .
$$

\section{REFERENCES}

ANDREwS, D., AND G. SOARES (2010): "Inference for Parameters Defined by Moment Inequalities Using Generalized Moment Selection Procedures,” Econometrica, 119-157. [1785]

Andrews, D., S. Berry, AND P. JiA (2006): "Confidence Regions for Parameters in Discrete

Games With Multiple Equilibria, With an Application to Discount Chain Store Location," Mimeo, Yale University. [1783,1794,1797]

BAJARI P., H. HONG, AND S. RYAN (2010): "Identification and Estimation of a Discrete Game of Complete Information," Econometrica (forthcoming). [1798]

BeCKERT, W., R. GRIFFITH, AND L. NeSheIm (2009): "Disaggregate Demand Elasticities at the Extensive and Intensive Margins: An Empirical Analysis of Store and Shopping Basket Choices," Mimeo, Institute for Fiscal Studies, London. [1788] 
Beresteanu, A., AND F. Molinari (2008): "Asymptotic Properties for a Class of Partially Identified Models," Econometrica, 76, 763-814. [1798]

CHERNOZHukOv, V., H. HONG, AND E. TAMER (2007): "Estimation and Confidence Regions for Parameter Sets in Econometric Models," Econometrica, 75, 1243-1284. [1785]

Ciliberto, F., AND E. TAMER (2009): "Market Structure and Multiple Equilibria in the Airline Markets," Econometrica, 77, 1791-1828. [1783,1794,1797]

Dekel, E., D. FudenberG, AND D. LeVINE (1993): "Payoff Information and Self-Confirming Equilibrium," Journal of Economic Theory, 89, 165-85. [1799]

De LoECKer, J., M. MelitZ, AND A. PAKES (2010): "Plant Location in the European Chemical Industry," Mimeo, Princeton University. [1803]

DuBIN, J., AND D. MCFADDEN (1984): "An Econometric Analysis of Residential Electric Appliance Holding and Consumption," Econometrica, 52, 345-362. [1787]

GAL-OR, E. (1997): "Exclusionary Equilibria in Health-Care Markets," Journal of Economics and Management Strategy, 6, 5-43. [1813]

Haile, P., AND E. TAMER (2003): "Inference With an Incomplete Model of English Auctions," Journal of Political Economy, 111, 1-51. [1784]

HANSEN, L. P., AND K. J. SinGLETON (1982): "Generalized Instrumental Variables Estimation of Nonlinear Rational Expectations Models," Econometrica, 50, 1269-1286. [1784]

HART, O., AND J. TIROLE (1990): "Vertical Integration and Market Foreclosure," Brookings Papers on Economic Activity: Microeconomics, 205-286. [1809]

HAUSMAn, J., J. ABREVAYA, AND F. SCOTT-MorTON (1998): "Misclassification of the Dependent Variable in a Discrete Choice Setting," Journal of Econometrics, 87, 239-269. [1795]

Ho, K. (2009): "Insurer-Provider Networks in the Medical Care Market," American Economic Review, 99, 393-430. [1784,1807,1808,1811,1816,1817,1819]

ISHII, J. (2008): "Compatability, Competition, and Investment in Network Industries: ATM Networks in the Banking Industry," Mimeo, GSB, Stanford University. [1802]

KATZ, M. (2007): “Supermarkets and Zoning Laws,” Unpublished Ph.D. Dissertation, Harvard University. [1785,1788]

KeANE, M., AND K. WOLPIN (2009): "Empirical Applications of Discrete Choice Dynamic Programming Models," Review of Economic Dynamics, 12, 1-22. [1795]

LEE, R., AND A. PAKES (2010): "Supplement to 'Alternative Models for Moment Inequalities,", Econometrica Supplemental Material, 79, http://www.econometricsociety.org/ecta/Supmat/ 7944_data data and programs.zip. [1813]

LUTTMER, E. (1996): "Asset Pricing in Economies With Frictions,” Econometrica, 64, 1439-1467. [1784]

MANSKI, C. (2004): “Measuring Expectations,” Econometrica, 72, 1329-1376. [1795]

MCAFEE, R., AND M. SCHWARTZ (1992): "Opportunism in Multilateral Vertical Contracting: Nondiscrimination, Exclusivity, and Uniformity," American Economic Review, 94, 210-230. [1809]

PAKes, A., J. Porter, K. Ho, AND J. IshiI (2006), "Moment Inequalities and Their Application," Mimeo, Harvard University. [1784,1792,1798,1800,1803,1811]

SAVAGE, L. (1954): The Foundations of Statistics. Hoboken, NJ: Wiley. [1791]

TAMER, E. (2003): "Incomplete Simultaneous Discrete Response With Multiple Equilibria," Review of Economic Studies, 70, 147-165. [1783,1794]

VARIAN, H. (1982): "The Nonparametric Approach to Demand Analysis," Econometrica, 50, 945-973. [1784]

Dept. of Economics, Harvard University, Littauer Room 117, Cambridge, MA 02138, U.S.A. and National Bureau of Economic Research; apakes@fas.harvard. edu. 